\title{
Linear Modal Analysis of L-Shaped Beam Structures
}

\author{
Fotios Georgiades $^{1 \mathrm{1a}^{*}}$, Jerzy Warminski ${ }^{1 \mathrm{~b}}$, Matthew, P., Cartmell ${ }^{2 \mathrm{c}}$ \\ ${ }^{1}$ Department of Applied Mechanics, Lublin University of Technology, Lublin, Poland \\ ${ }^{2}$ School of Engineering, University of Glasgow, Glasgow, UK \\ af.georgiadis@pollub.pl, ${ }^{\text {bj.warminski@pollub.pl, }}{ }^{\mathrm{c}}$ matthew.cartmell@ glasgow.ac.uk
}

*Corresponding author. Address: Lublin University of Technology, Department of Applied

Mechanics, Nadbystrzycka 36, 20-618 Lublin, Poland. Phone: +48-81-53-84-144. Fax:+48-81-53-84-

205.

\begin{abstract}
In this article a theoretical linear modal analysis of Euler-Bernoulli L-shaped beam structures is performed by solving two sets of coupled partial differential equations of motion. The first set, with two equations, corresponds to in-plane bending motions whilst the second set with four equations corresponds to out-of-plane motions with bending and torsion. The case is also shown of a single cantilever beam taking into account rotary inertia terms. At first for the case of examination of the results for the L-shaped beam structure, an individual modal analysis is presented for four selected beams which will be used for modelling an L-shaped beam structure; in order to investigate the influence of rotary inertia terms and shear effects. Then, a theoretical and numerical modal analysis is performed for four models of the L-shaped beam structure consisting of two sets of beams, in order to examine the effect of the orientation of the secondary beam (oriented in two ways) and also shear effects. The comparison of theoretical and finite element simulations shows a good agreement for both in-plane and out-of-plane motions, which validates the theoretical analysis. This work is essential to make progress with new investigations into the nonlinear equations for the L-shaped beam structures within Nonlinear Normal Mode theory.
\end{abstract}

Keywords: L-shaped beam structure, modal analysis, elastic continua dynamics, Euler-Bernoulli beams, Autoparametric structure

\section{Introduction}

There has been continuous interest in the dynamics of L-shaped coupled structures exhibiting nonlinearities and autoparametric coupling since the 1960s. Roberts and Cartmell in [1,2] studied certain autoparametric resonances within an L-shaped beam structure, and Balachandran and Nayfeh performed nonlinear modal analysis considering only in-plane motions [3]. Warminski et al. in [4] formulated the third order partial differential nonlinear equations for an L-shaped beam structure with different flexibilities in the two orthogonal directions, without taking into account rotary inertia effects. Ozonato et al. studied post-buckled chaotic vibrations of an L-shaped beam structure considering only in-plane bending nonlinear motions [5]. Nayfeh and Pai [6], studied many cases of autoparametric excitations of beams. In Georgiades et al. [7] the linear equations of motion have been derived for an L-shaped beam structure, considering the inextensionality conditions and rotary inertia terms. That study demonstrated well separated in-plane and out-of-plane motions, which has also been shown with Abaqus Finite Element (FE) simulations. Also the necessity for rotary inertia terms in outof-plane bending was confirmed. In the case that the rotary inertia terms are neglected then the torsional equation of the secondary beam becomes uncoupled from the rest of the out-of-plane motions, and modal analysis for torsion of the secondary beam leads to very different results from the numerical simulations. 
In this article we start from the equations of motion and then a theoretical linear modal analysis of the L-shaped Euler Bernoulli beam structure is undertaken considering in-plane and out-ofplane motions. Using two sets of dimensions four models are built in the Abaqus software in order to compare the theoretical with the numerical results. Initially FE models are built and theoretical and numerical modal analyses are undertaken for each one of the individual beams to examine shear and rotary inertia effects in the dimensions of the beams that are considered. Finally, the results for the Lshaped beam structure of the analytical model are compared with the numerical results to validate the theoretical linear modal analysis.

\section{Theory}

\subsection{Equations of motion}

Beams constructed of isotropic material are considered, with constant cross section with respect to the longitudinal direction (Fig.1). The equations of motion were derived in [7] and are given by,

In-plane motions (bending),

$$
\begin{gathered}
-m_{1} \ddot{v}_{1}-D_{\zeta_{1}} v_{1}^{I V}+\delta_{4} I_{\zeta_{1}} \ddot{v}_{1}^{\prime \prime}=0, \\
-m_{2}\left(\ddot{w}_{2}-s_{2} \ddot{v}_{1 C}^{\prime}+\ddot{u}_{1 C}\right)-D_{\eta_{2}} w_{2}^{I V}+\delta_{5} I_{\eta_{2}} \ddot{w}_{2}^{\prime \prime}=0,
\end{gathered}
$$

with boundary conditions,

$$
\begin{gathered}
v_{1}(0, t)=0, \quad v_{1}^{\prime}(0, t)=0, \\
-\delta_{4} I_{\zeta_{1}} \ddot{v}_{1}^{\prime}\left(l_{1}, t\right)+D_{\zeta_{1}} v_{1}^{\prime \prime \prime}\left(l_{1}, t\right)-l_{2} m_{2} \ddot{v}_{1}\left(l_{1}, t\right)=0, \\
-D_{\zeta_{1}} v_{1}^{\prime \prime}\left(l_{1}, t\right)-D_{\eta_{2}} w_{2}^{\prime \prime}(0, t)+I_{\eta_{2}} \delta_{4} \delta_{5} l_{2} \ddot{v}_{1}^{\prime}\left(l_{1}, t\right)-I_{\eta_{2}} \delta_{5} \ddot{w}_{2}\left(l_{2}, t\right)+ \\
+\frac{I_{\eta_{2}}}{m_{2}} \delta_{4} \delta_{5}\left\{-D_{\eta_{2}}\left[w_{2}^{I V}\left(l_{2}, t\right)-w_{2}^{I V}(0, t)\right]-I_{\eta_{2}} \delta_{5} \ddot{w}_{2}^{\prime \prime}(0, t)\right\}=0, \\
w_{2}(0, t)=0, \quad c \\
w_{2}^{\prime}(0, t)=0, \\
D_{\eta_{2}} w_{2}^{\prime \prime \prime}\left(l_{2}, t\right)+\delta_{5} I_{\eta_{2}}\left(-\ddot{w}_{2}^{\prime}+\ddot{v}_{1}^{\prime}\left(l_{1}, t\right)\right)=0, \\
w_{2}^{\prime \prime}\left(l_{2}, t\right)=0 .
\end{gathered}
$$

Out-of-plane motions,

bending,

$$
\begin{array}{r}
-m_{1} \ddot{w}_{1}+\delta_{1} I_{\eta_{1}} \ddot{w}_{1}^{\prime \prime}-D_{\eta_{1}} w_{1}^{I V}=0 \\
-m_{2}\left(\ddot{v}_{2}+s_{2} \ddot{\varphi}_{1 C}+\ddot{w}_{1 C}\right)+\delta_{2} I_{\zeta_{2}} \ddot{v}_{2}^{\prime \prime}-D_{\zeta_{2}} v_{2}^{I V}=0,
\end{array}
$$

torsion,

$$
\begin{gathered}
-I_{\xi_{1}} \ddot{\varphi}_{1}+D_{\xi_{1}} \varphi_{1}^{\prime \prime}=0, \\
-I_{\xi_{2}}\left(\ddot{\varphi}_{2}-\delta_{1} \delta_{3} \ddot{w}_{1 C}^{\prime}\right)+D_{\xi_{2}} \varphi_{2}^{\prime \prime}=0,
\end{gathered}
$$

with boundary conditions,

$$
\begin{aligned}
& w_{1}(0, t)=0, \quad w_{1}^{\prime}(0, t)=0, \\
& -\delta_{1} I_{\eta_{1}} \ddot{w}_{1}^{\prime}\left(l_{1}, t\right)+D_{\eta_{1}} w_{1}^{\prime \prime \prime}\left(l_{1}, t\right)+\delta_{2} I_{\zeta_{2}} \ddot{\varphi}_{1}\left(l_{1}, t\right)-D_{\zeta_{2}} v_{2}^{\prime \prime \prime}(0, t)=0, \\
& -D_{\eta_{1}} w_{1}^{\prime \prime}\left(l_{1}, t\right)-\delta_{1} \delta_{3} D_{\xi_{2}} \varphi_{2}^{\prime}(0, t)=0 \text {, } \\
& \varphi_{1}(0, t)=0 \text {, } \\
& -D_{\xi_{1}} \varphi_{1}^{\prime}\left(l_{1}, t\right)+\delta_{2} l_{2} I_{\zeta_{2}} \ddot{\varphi}_{1}\left(l_{1}, t\right)+\delta_{2} I_{\zeta_{2}} \ddot{v}_{2}\left(l_{2}, t\right)+D_{\zeta_{2}} v_{2}^{\prime \prime}(0, t)+\frac{I_{\zeta_{2}}^{2}}{m_{2}} \delta_{2} \ddot{v}_{2}^{\prime \prime}(0, t)+ \\
& +\delta_{2} \frac{I_{\zeta_{2}} D_{\zeta_{2}}}{m_{2}}\left(v_{2}^{I V}\left(l_{2}, t\right)-v_{2}^{I V}(0, t)\right)=0 \\
& v_{2}(0, t)=0, \quad v_{2}^{\prime}(0, t)=0, \\
& -\delta_{2} I_{\zeta_{2}}\left(\ddot{v}_{2}^{\prime}\left(l_{2}, t\right)+\ddot{\varphi}_{1}\left(l_{1}, t\right)\right)+D_{\zeta_{2}} v_{2}^{\prime \prime \prime}\left(l_{2}, t\right)=0, \quad v_{2}^{\prime \prime}\left(l_{2}, t\right)=0, \\
& \varphi_{2}(0, t)=0, \quad \varphi_{2}^{\prime}\left(l_{2}, t\right)=0 .
\end{aligned}
$$


The coordinates $u_{1}, v_{l}, w_{l}$, denote the displacements of a selected arbitrary point of the primary (horizontal) beam in the absolute $\mathrm{X}_{1}, \mathrm{Y}_{1}, \mathrm{Z}_{1}$ coordinate set, and $u_{2}, v_{2}, w_{2}$, are the displacements of the secondary (vertical) beam in the local coordinate set $\mathrm{X}_{2}, \mathrm{Y}_{2}, \mathrm{Z}_{2}$ fixed to the centre of a cross-section at point C (see Fig.1), and $\varphi_{1}, \varphi_{2}$ are angles of rotation about the $\xi_{1}$ and $\xi_{2}$ axes respectively. The dot and prime mean derivatives with respect to time and space, respectively.

The Kronecker delta function is used in order to model the effect of rotary inertia terms in the final equations.

The following transformations, are applied

$$
W_{2}=w_{2}-s_{2} v_{1 C}^{\prime}+u_{1 C}, V_{2}=v_{2}+s_{2} \varphi_{1 C}+w_{1 C}, \phi_{2}=\varphi_{2}-w_{1 C}^{\prime},
$$

to express the local displacements and rotations of the secondary beam in terms of global displacements and rotations. For in-plane bending the rotary inertia terms have a very small influence and can be neglected. Therefore using $\delta_{4}=\delta_{5}=0$, and considering the inertia terms for out-of-plane bending $\delta_{1}=\delta_{2}=\delta_{3}=1$, and also using eq. (13a-c) in eq. (1-12), then the equations of motion and the boundary conditions are given by,

a) In-plane motions,

with boundary conditions,

$$
m_{1} \ddot{v}_{1}+D_{\zeta_{1}} v_{1}^{I V}=0, \quad m_{2} \ddot{W}_{2}+D_{\eta_{2}} W_{2}^{I V}=0,
$$

$$
\begin{array}{r}
v_{1}(0, t)=0, \quad v_{1}^{\prime}(0, t)=0, \quad D_{\zeta_{1}} v_{1}^{\prime \prime \prime}\left(l_{1}, t\right)=l_{2} m_{2} \ddot{v}_{1}\left(l_{1}, t\right), D_{\zeta_{1}} v_{1}^{\prime \prime}\left(l_{1}, t\right)=-D_{\eta_{2}} W_{2}^{\prime \prime}(0, t), \\
W_{2}(0, t)=0^{1}, \quad W_{2}^{\prime}(0, t)=-v_{1}^{\prime}\left(l_{1}, t\right), W_{2}^{\prime \prime}\left(l_{2}, t\right)=0, \quad W_{2}^{\prime \prime \prime}\left(l_{2}, t\right)=0, \quad(15 \mathrm{a}-\mathrm{h}),
\end{array}
$$

b) Out-of-plane motions,

$$
\begin{aligned}
& m_{1} \ddot{w}_{1}-I_{\eta_{1}} \ddot{w}_{1}^{\prime \prime}+D_{\eta_{1}} w_{1}^{I V}=0, \quad m_{2} \ddot{V}_{2}-I_{\zeta_{2}} \ddot{V}_{2}^{\prime \prime}+D_{\zeta_{2}} V_{2}^{I V}=0, \\
& I_{\xi_{1}} \ddot{\varphi}_{1}-D_{\xi_{1}} \varphi_{1}^{\prime \prime}=0, \quad I_{\xi_{2}} \ddot{\phi}_{2}-D_{\xi_{2}} \phi_{2}^{\prime \prime}=0,
\end{aligned}
$$

with boundary conditions,

$$
\begin{gathered}
w_{1}(0, t)=0, \quad w_{1}^{\prime}(0, t)=0, \\
-I_{\eta_{1}} \ddot{w}_{1}^{\prime}\left(l_{1}, t\right)+D_{\eta_{1}} w_{1}^{\prime \prime \prime}\left(l_{1}, t\right)+I_{\zeta_{2}} \ddot{\varphi}_{1}\left(l_{1}, t\right)-D_{\zeta_{2}} V_{2}^{\prime \prime \prime}(0, t)=0, \\
D_{\eta_{1}} w_{1}^{\prime \prime}\left(l_{1}, t\right)+D_{\xi_{2}} \phi_{2}^{\prime}(0, t)=0, \\
\varphi_{1}(0, t)=0, \\
D_{\xi_{1}} \varphi_{1}^{\prime}\left(l_{1}, t\right)+I_{\zeta_{2}} \ddot{w}_{1}\left(l_{1}, t\right)-I_{\zeta_{2}} \ddot{V}_{2}\left(l_{2}, t\right)-D_{\zeta_{2}} V_{2}^{\prime \prime}(0, t)-\frac{I_{\zeta_{2}}^{2}}{m_{2}} \ddot{V}_{2}^{\prime \prime}(0, t)- \\
-\frac{I_{\zeta_{2}} D_{\zeta_{2}}}{m_{2}}\left(V_{2}^{I V}\left(l_{2}, t\right)-V_{2}^{I V}(0, t)\right)=0, \\
V_{2}^{\prime}(0, t)=\varphi_{1}\left(l_{1}, t\right), \\
V_{2}(0, t)=w_{1}\left(l_{1}, t\right), \quad V_{2}^{\prime \prime}\left(l_{2}, t\right)=0, \\
-I_{\zeta_{2}} \ddot{V}_{2}^{\prime}\left(l_{2}, t\right)+D_{\zeta_{2}} V_{2}^{\prime \prime \prime}\left(l_{2}, t\right)=0, \quad
\end{gathered}
$$

\footnotetext{
${ }^{1}$ In this boundary condition, $W_{2}(0, t)=u_{1 C}$, but the axial displacement is of second order due to the inextensionality condition, therefore it is neglected for the first order linear problem.
} 


$$
\phi_{2}(0, t)=-w_{1}^{\prime}\left(l_{1}, t\right), \quad \phi_{2}^{\prime}\left(l_{2}, t\right)=0 .
$$

In the next section a theoretical modal analysis of the equations of motion is performed (eq. 14-20).

\subsection{Modal analysis}

\subsubsection{In-plane motion}

Using the method of separation of variables in space and time, by means of,

$$
v_{1}\left(s_{1}, t\right)=Y_{v}\left(s_{1}\right) \cdot a_{v}(t), W_{2}\left(s_{2}, t\right)=Y_{W}\left(s_{2}\right) \cdot a_{W}(t),
$$

the equations of in-plane motion (eq. 14) take the form,

$$
\begin{array}{cc}
Y_{v}^{I V}\left(s_{1}\right)-\mu_{v}^{4} Y_{v}\left(s_{1}\right)=0, & Y_{W}^{I V}\left(s_{1}\right)-\mu_{W}^{4} Y_{W}\left(s_{1}\right)=0, \\
\frac{\ddot{a}_{v}(t)}{a_{v}(t)}=\frac{\ddot{a}_{W}(t)}{a_{W}(t)}=-\omega_{i n}^{2}=-\frac{D_{\zeta_{1}}}{m_{1}} \mu_{v}^{4}=-\frac{D_{\eta_{2}}}{m_{2}} \mu_{W}^{4},
\end{array}
$$

The equality on the left hand side of equation (22c) arises from the fact that for linear modes the two beams must execute synchronous motion, therefore they must have the same period. Using equations (21), (22) and also eq. (14a), the boundary conditions of eq. (15) take the form,

$$
\begin{gathered}
Y_{v}(0)=0, \quad Y_{v}^{\prime}(0)=0, \quad Y_{v}^{\prime \prime \prime}\left(l_{1}\right)=-\frac{l_{2} m_{2}}{m_{1}} Y_{v}^{I V}\left(l_{1}\right), \\
D_{\zeta_{1}} Y_{v}^{\prime \prime}\left(l_{1}, t\right)=-D_{\eta_{2}} Y_{W}^{\prime \prime}(0), \quad Y_{W}(0)=0 \\
Y_{W}^{\prime}(0)=-Y_{v}^{\prime}\left(l_{1}\right), \quad Y_{W}^{\prime \prime}\left(l_{2}\right)=0, \quad Y_{W}^{\prime \prime \prime}\left(l_{2}\right)=0 .
\end{gathered}
$$

The mode shapes can be determined from the solution of the ordinary differential equations of $(22 \mathrm{a}, \mathrm{b})$, which is a trivial problem and those solutions are given by,

$$
\begin{aligned}
& Y_{v}\left(s_{1}\right)=\mathrm{C}_{1} \sin \left(\mu_{v} s_{1}\right)+\mathrm{C}_{2} \cos \left(\mu_{v} s_{1}\right)+\mathrm{C}_{3} \sinh \left(\mu_{v} s_{1}\right)+\mathrm{C}_{4} \cosh \left(\mu_{v} s_{1}\right), \\
& Y_{W}\left(s_{1}\right)=\mathrm{D}_{1} \sin \left(\mu_{W} s_{2}\right)+\mathrm{D}_{2} \cos \left(\mu_{W} s_{2}\right)+\mathrm{D}_{3} \sinh \left(\mu_{W} s_{2}\right)+\mathrm{D}_{4} \cosh \left(\mu_{W} s_{2}\right),
\end{aligned}
$$

The constants in these equations are determined as functions of $\mathrm{C}_{1}$ and are given in Appendix-A by means of some appropriate algebraic manipulations and using the boundary conditions (eq. 23a-c,e-h) and eq. $(24 a, b)$. These constants are given by,

$$
\begin{gathered}
\mathrm{C}_{2}=\mathrm{C}_{1}\left\{\frac{-\cos \left(\mu_{v} l_{1}\right)-\cosh \left(\mu_{v} l_{1}\right)+\frac{m_{2} l_{2} \mu_{v}}{m_{1}}\left[\sin \left(\mu_{v} l_{1}\right)-\sinh \left(\mu_{v} l_{1}\right)\right]}{\sinh \left(\mu_{v} l_{1}\right)-\sin \left(\mu_{v} l_{1}\right)+\frac{m_{2} l_{2} \mu_{v}}{m_{1}}\left[\cosh \left(\mu_{v} l_{1}\right)-\cos \left(\mu_{v} l_{1}\right)\right]}\right\}=\mathrm{C}_{1} \mathrm{C}_{L}, \\
\mathrm{C}_{4}=-\mathrm{C}_{1}\left\{\frac{-\cos \left(\mu_{v} l_{1}\right)-\cosh \left(\mu_{v} l_{1}\right)+\frac{m_{2} l_{2} \mu_{v}}{m_{1}}\left[\sin \left(\mu_{v} l_{1}\right)-\sinh \left(\mu_{v} l_{1}\right)\right]}{\sinh \left(\mu_{v} l_{1}\right)-\sin \left(\mu_{v} l_{1}\right)+\frac{m_{2} l_{2} \mu_{v}}{m_{1}}\left[\cosh \left(\mu_{v} l_{1}\right)-\cos \left(\mu_{v} l_{1}\right)\right]}\right\}=-\mathrm{C}_{1} \mathrm{C}_{L}, \\
D_{1}=-\frac{\mu_{v}\left\{\cos \left(\mu_{v} l_{1}\right)-\cosh \left(\mu_{v} l_{1}\right)+\mathrm{C}_{L}\left[-\sin \left(\mu_{v} l_{1}\right)-\sinh \left(\mu_{v} l_{1}\right)\right]\right\}}{\mu_{W}\left\{1+\frac{\sin \left(\mu_{W} l_{2}\right)}{\sinh \left(\mu_{W} l_{2}\right)}+\frac{\left[\cos \left(\mu_{W} l_{2}\right) \sinh \left(\mu_{W} l_{2}\right)-\sin \left(\mu_{W} l_{2}\right) \cosh \left(\mu_{W} l_{2}\right)\right]\left[\cos \left(\mu_{W} l_{2}\right)+\cosh \left(\mu_{W} l_{2}\right)\right]}{\sinh \left(\mu_{W} l_{2}\right)\left[\sin \left(\mu_{W} l_{2}\right) \sinh \left(\mu_{W} l_{2}\right)+\cos \left(\mu_{W} l_{2}\right) \cosh \left(\mu_{W} l_{2}\right)+1\right]} \mathrm{C}_{1},\right.}
\end{gathered}
$$

by using equation (26a) and equations (A.5,7,8) the other constants for equation (24b) are given as,

$$
\mathrm{D}_{2}=-\left[\frac{\sinh \left(\mu_{W} l_{2}\right) \cos \left(\mu_{W} l_{2}\right)-\sin \left(\mu_{W} l_{2}\right) \cosh \left(\mu_{W} l_{2}\right)}{\sin \left(\mu_{W} l_{2}\right) \sinh \left(\mu_{W} l_{2}\right)+\cos \left(\mu_{W} l_{2}\right) \cosh \left(\mu_{W} l_{2}\right)+1}\right] \times
$$




$$
\begin{aligned}
& \times\left[\frac{\mu_{v}\left\{\cos \left(\mu_{v} l_{1}\right)-\cosh \left(\mu_{v} l_{1}\right)+\mathrm{C}_{L}\left[-\sin \left(\mu_{v} l_{1}\right)-\sinh \left(\mu_{v} l_{1}\right)\right]\right\}}{\mu_{W}\left\{1+\frac{\sin \left(\mu_{W} l_{2}\right)}{\sinh \left(\mu_{W} l_{2}\right)}+\frac{\left[\cos \left(\mu_{W} l_{2}\right) \sinh \left(\mu_{W} l_{2}\right)-\sin \left(\mu_{W} l_{2}\right) \cosh \left(\mu_{W} l_{2}\right)\right]\left[\cos \left(\mu_{W} l_{2}\right)+\cosh \left(\mu_{W} l_{2}\right)\right]}{\sinh \left(\mu_{W} l_{2}\right)\left[\sin \left(\mu_{W} l_{2}\right) \sinh \left(\mu_{W} l_{2}\right)+\cos \left(\mu_{W} l_{2}\right) \cosh \left(\mu_{W} l_{2}\right)+1\right]}\right\}}\right] \mathrm{C}_{1}, \\
& \mathrm{D}_{3}=\left\{\frac{\sin \left(\mu_{W} l_{2}\right)}{\sinh \left(\mu_{W} l_{2}\right)}+\frac{\left[\cos \left(\mu_{W} l_{2}\right) \sinh \left(\mu_{W} l_{2}\right)-\sin \left(\mu_{W} l_{2}\right) \cosh \left(\mu_{W} l_{2}\right)\right]\left[\cos \left(\mu_{W} l_{2}\right)+\cosh \left(\mu_{W} l_{2}\right)\right]}{\sinh \left(\mu_{W} l_{2}\right)\left[\sin \left(\mu_{W} l_{2}\right) \sinh \left(\mu_{W} l_{2}\right)+\cos \left(\mu_{W} l_{2}\right) \cosh \left(\mu_{W} l_{2}\right)+1\right]}\right\} \times
\end{aligned}
$$

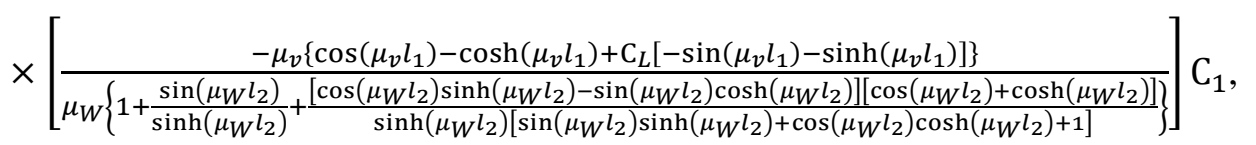

$$
\begin{aligned}
& \mathrm{D}_{4}=\left[\frac{\sinh \left(\mu_{W} l_{2}\right) \cos \left(\mu_{W} l_{2}\right)-\sin \left(\mu_{W} l_{2}\right) \cosh \left(\mu_{W} l_{2}\right)}{\sin \left(\mu_{W} l_{2}\right) \sinh \left(\mu_{W} l_{2}\right)+\cos \left(\mu_{W} l_{2}\right) \cosh \left(\mu_{W} l_{2}\right)+1}\right] \times \\
& \times\left[\frac{\mu_{v}\left\{\cos \left(\mu_{v} l_{1}\right)-\cosh \left(\mu_{v} l_{1}\right)+C_{L}\left[-\sin \left(\mu_{v} l_{1}\right)-\sinh \left(\mu_{v} l_{1}\right)\right]\right\}}{\mu_{W}\left\{1+\frac{\sin \left(\mu_{W} l_{2}\right)}{\sinh \left(\mu_{W} l_{2}\right)}+\frac{\left[\cos \left(\mu_{W} l_{2}\right) \sinh \left(\mu_{W} l_{2}\right)-\sin \left(\mu_{W} l_{2}\right) \cosh \left(\mu_{W} l_{2}\right)\right]\left[\cos \left(\mu_{W} l_{2}\right)+\cosh \left(\mu_{W} l_{2}\right)\right]}{\sinh \left(\mu_{W} l_{2}\right)\left[\sin \left(\mu_{W} l_{2}\right) \sinh \left(\mu_{W} l_{2}\right)+\cos \left(\mu_{W} l_{2}\right) \cosh \left(\mu_{W} l_{2}\right)+1\right]}\right]}\right] \mathrm{C}_{1} \cdot(26 \mathrm{~d})
\end{aligned}
$$

The natural frequencies can be determined through equation $(23 \mathrm{~d})$, and $\mu_{v}$ which are defined by the roots of a transcedental equation. This equation originates from the boundary condition equation (23d) and equations $(24 a, b)$ and is given by,

$$
D_{\zeta_{1}} \mu_{v}^{2}\left[-\mathrm{C}_{1} \sin \left(\mu_{v} l_{1}\right)-\mathrm{C}_{2} \cos \left(\mu_{v} l_{1}\right)+\mathrm{C}_{3} \sinh \left(\mu_{v} l_{1}\right)+\mathrm{C}_{4} \cosh \left(\mu_{v} l_{1}\right)\right]=D_{\eta_{2}} \mu_{W}^{2}\left[\mathrm{D}_{2}-\mathrm{D}_{4}\right]
$$

by also using eq. $(25,26)$ and some manipulations, it emerges that,

$$
\begin{aligned}
& \sqrt[4]{\frac{m_{1} D_{\zeta_{1}}^{3}}{m_{2} D_{\eta_{2}}^{3}}} \times\left\{-\sin \left(\mu_{v} l_{1}\right)-\sinh \left(\mu_{v} l_{1}\right)-\left[\cos \left(\mu_{v} l_{1}\right)+\cosh \left(\mu_{v} l_{1}\right)\right] \times\right. \\
& \left.\times\left[\frac{-\cos \left(\mu_{v} l_{1}\right)-\cosh \left(\mu_{v} l_{1}\right)+\frac{m_{2} l_{2} \mu_{v}}{m_{1}}\left(\sin \left(\mu_{v} l_{1}\right)-\sinh \left(\mu_{v} l_{1}\right)\right)}{\sinh \left(\mu_{v} l_{1}\right)-\sin \left(\mu_{v} l_{1}\right)+\frac{m_{2} l_{2} \mu_{v}}{m_{1}}\left(\cosh \left(\mu_{v} l_{1}\right)-\cos \left(\mu_{v} l_{1}\right)\right)}\right]\right\} \times \\
& \times\left\{1+\frac{\sin \left(\frac{\mu_{v} l_{2}}{\gamma}\right)}{\sinh \left(\frac{\mu_{v} l_{2}}{\gamma}\right)}+\frac{\left[\cos \left(\frac{\mu_{v} l_{2}}{\gamma}\right) \sinh \left(\frac{\mu_{v} l_{2}}{\gamma}\right)-\sin \left(\frac{\mu_{v} l_{2}}{\gamma}\right) \cosh \left(\frac{\mu_{v} l_{2}}{\gamma}\right)\right]\left[\cos \left(\frac{\mu_{v} l_{2}}{\gamma}\right)+\cosh \left(\frac{\mu_{v} l_{2}}{\gamma}\right)\right]}{\sinh \left(\frac{\mu_{v} l_{2}}{\gamma}\right)\left[\sin \left(\frac{\mu_{v} l_{2}}{\gamma}\right) \sinh \left(\frac{\mu_{v} l_{2}}{\gamma}\right)+\cos \left(\frac{\mu_{v} l_{2}}{\gamma}\right) \cosh \left(\frac{\mu_{v} l_{2}}{\gamma}\right)+1\right]}\right\} \times \\
& \times\left[\sin \left(\frac{\mu_{v} l_{2}}{\gamma}\right) \sinh \left(\frac{\mu_{v} l_{2}}{\gamma}\right)+\cos \left(\frac{\mu_{v} l_{2}}{\gamma}\right) \cosh \left(\frac{\mu_{v} l_{2}}{\gamma}\right)+1\right]+ \\
& +2\left[\sinh \left(\frac{\mu_{v} l_{2}}{\gamma}\right) \cos \left(\frac{\mu_{v} l_{2}}{\gamma}\right)-\sin \left(\frac{\mu_{v} l_{2}}{\gamma}\right) \cosh \left(\frac{\mu_{v} l_{2}}{\gamma}\right)\right] \times \\
& \times\left\{\cos \left(\mu_{v} l_{1}\right)-\cosh \left(\mu_{v} l_{1}\right)-\left[\sin \left(\mu_{v} l_{1}\right)+\sinh \left(\mu_{v} l_{1}\right)\right] \times\right. \\
& \left.\times\left[\frac{-\cos \left(\mu_{v} l_{1}\right)-\cosh \left(\mu_{v} l_{1}\right)+\frac{m_{2} l_{2} \mu_{v}}{m_{1}}\left(\sin \left(\mu_{v} l_{1}\right)-\sinh \left(\mu_{v} l_{1}\right)\right)}{\sinh \left(\mu_{v} l_{1}\right)-\sin \left(\mu_{v} l_{1}\right)+\frac{m_{2} l_{2} \mu_{v}}{m_{1}}\left(\cosh \left(\mu_{v} l_{1}\right)-\cos \left(\mu_{v} l_{1}\right)\right)}\right]\right\}=0,
\end{aligned}
$$

with,

$$
\gamma=\frac{\mu_{v}}{\mu_{W}}=\sqrt[4]{\frac{m_{1} D_{\eta_{2}}}{m_{2} D_{\zeta_{1}}}}
$$

It should be noted that it is very difficult to obtain explicit formulae for the roots of the transcedental equation (28). Considering parameters for a given L-shaped beam structure, then the roots of this equation can be found easily by examining the zero crossings of the plot of this equation with respect to $\mu_{v}$. 


\subsubsection{Out-of-plane motion}

Using the method of separation of variables in space and time for out-of-plane motion, by means of,

$$
\begin{gathered}
w_{1}\left(s_{1}, t\right)=Y_{w}\left(s_{1}\right) \cdot h_{w}(t), V_{2}\left(s_{2}, t\right)=Y_{V}\left(s_{2}\right) \cdot h_{V}(t), \\
\varphi_{1}\left(s_{1}, t\right)=Y_{\varphi}\left(s_{1}\right) \cdot h_{\varphi}(t), \phi_{2}\left(s_{2}, t\right)=Y_{\Phi}\left(s_{2}\right) \cdot h_{\Phi}(t),
\end{gathered}
$$

equations (16) then take the form,

$$
\begin{gathered}
Y_{w}^{I V}+\eta_{w}^{2}\left(\frac{I_{\eta_{1}}}{m_{1}}\right) Y_{w}^{\prime \prime}-\eta_{w}^{2} Y_{w}=0, Y_{V}^{I V}+\eta_{V}^{2}\left(\frac{I_{\zeta_{2}}}{m_{2}}\right) Y_{V}^{\prime \prime}-\eta_{V}^{2} Y_{V}=0, \\
Y_{\varphi}^{\prime \prime}+\eta_{\varphi}^{2} Y_{\varphi}=0, Y_{\Phi}^{\prime \prime}+\eta_{\Phi}^{2} Y_{\Phi}=0, \\
h_{w}(t)=h_{V}(t)=h_{\varphi}(t)=h_{\Phi}(t)=h(t), \\
\frac{\ddot{h}(t)}{h(t)}=-\omega_{o u t}^{2}=-\frac{D_{\eta_{1}}}{m_{1}} \eta_{w}^{2}=-\frac{D \zeta_{2}}{m_{2}} \eta_{V}^{2}=-\frac{D_{\xi_{1}}}{I_{\xi_{1}}} \eta_{\varphi}^{2}=-\frac{D \xi_{2}}{I_{\xi_{2}}} \eta_{\Phi}^{2},
\end{gathered}
$$

Similarly to the case of the in-plane motions, equation (31e) arises from the fact that for linear modes the two beams must execute synchronous motions with the same period. Consequently the solution of the differential equation in the time domain will be the same for all the variables. Therefore, using equations $(30,31)$, the boundary conditions (17-20) take the form,

$$
\begin{gathered}
Y_{w}(0)=0, \quad Y_{w}^{\prime}(0)=0, \\
\omega_{\text {out }}^{2} I_{\eta_{1}} Y_{w}^{\prime}\left(l_{1}\right)+D_{\eta_{1}} Y_{w}^{\prime \prime \prime}\left(l_{1}, t\right)-I_{\zeta_{2}} \omega_{\text {out }}^{2} Y_{\varphi}\left(l_{1}\right)-D_{\zeta_{2}} Y_{V}^{\prime \prime \prime}(0)=0, \\
D_{\eta_{1}} Y_{w}^{\prime \prime}\left(l_{1}\right)+D_{\xi_{2}} Y_{\Phi}^{\prime}(0)=0, \\
Y_{\varphi}(0)=0, \\
D_{\xi_{1}} Y_{\varphi}^{\prime}\left(l_{1}\right)-\omega_{\text {out }}^{2} I_{\zeta_{2}} Y_{w}\left(l_{1}\right)+I_{\zeta_{2}} \omega_{\text {out }}^{2} Y_{V}\left(l_{2}\right)-D_{\zeta_{2}} Y_{V}^{\prime \prime}(0)+\omega_{\text {out }}^{2} \frac{I_{\zeta_{2}}^{2}}{m_{2}} Y_{V}^{\prime \prime}(0)- \\
-\frac{I_{\zeta_{2}} D_{\zeta_{2}}}{m_{2}}\left(Y_{V}^{I V}\left(l_{2}\right)-Y_{V}^{I V}(0)\right)=0, \\
Y_{V}^{\prime}(0)=Y_{\varphi}\left(l_{1}\right), \\
Y_{w}\left(l_{1}\right), \\
\omega_{\text {out }}^{2} I_{\zeta_{2}} Y_{V}^{\prime}\left(l_{2}\right)+D_{\zeta_{2}} Y_{V}^{\prime \prime \prime}\left(l_{2}\right)=0, \\
Y_{\Phi}(0)=-Y_{w}^{\prime \prime}\left(l_{1}\right),
\end{gathered}
$$

As shown in Appendix-B the general solutions of equations (31a,b) are given by,

$$
\begin{gathered}
Y_{w}\left(s_{1}\right)=E_{1} \sin \left(d_{1} s_{1}\right)+E_{2} \cos \left(d_{1} s_{1}\right)+E_{3} \sinh \left(d_{2} s_{1}\right)+E_{4} \cosh \left(d_{2} s_{1}\right), \\
Y_{V}\left(s_{2}\right)=F_{1} \sin \left(f_{1} s_{2}\right)+F_{2} \cos \left(f_{1} s_{2}\right)+F_{3} \sinh \left(f_{2} s_{2}\right)+F_{4} \cosh \left(f_{2} s_{2}\right),
\end{gathered}
$$

with, 


$$
\begin{gathered}
d_{1}=\sqrt{\frac{\eta_{w}^{2}\left(\frac{I \eta_{1}}{m_{1}}\right)+\sqrt{\eta_{w}^{4}\left(\frac{I_{\eta_{1}}}{m_{1}}\right)^{2}+4 \eta_{w}^{2}}}{4}, d_{2}=\sqrt{\frac{-\eta_{w}^{2}\left(\frac{I \eta_{1}}{m_{1}}\right)+\sqrt{\eta_{w}^{4}\left(\frac{I \eta_{1}}{m_{1}}\right)^{2}+4 \eta_{w}^{2}}}{4}},} \\
f_{1}=\sqrt{\frac{\eta_{V}^{2}\left(\frac{I \zeta_{2}}{m_{2}}\right)+\sqrt{\eta_{V}^{4}\left(\frac{I_{2}}{m_{2}}\right)^{2}+4 \eta_{V}^{2}}}{4}}, f_{2}=\sqrt{\frac{\left.-\eta_{V}^{2}\left(\frac{I}{m_{2}}\right)_{2}\right)+\sqrt{\eta_{V}^{4}\left(\frac{I_{2}}{m_{2}}\right)^{2}+4 \eta_{V}^{2}}}{4}} .
\end{gathered}
$$

The general solutions of equations $(31 \mathrm{c}, \mathrm{d})$ are given by,

$$
\begin{aligned}
& Y_{\varphi}\left(s_{1}\right)=K_{1} \sin \left(\eta_{\varphi} s_{1}\right)+K_{2} \cos \left(\eta_{\varphi} s_{1}\right), \\
& Y_{\Phi}\left(s_{2}\right)=M_{1} \sin \left(\eta_{\Phi} s_{2}\right)+M_{2} \cos \left(\eta_{\Phi} s_{2}\right)
\end{aligned}
$$

The mode shapes for out-of-plane motion are defined by equations (36) and (38), and the general solution constants have been determined with respect to $E_{1}$ through the boundary conditions (32-35), as explained in Appendix-C. These constants are given by,

$$
\begin{gathered}
E_{1}=E_{2} q_{3}, \quad E_{3}=-\frac{d_{1}}{d_{2}} E_{2} q_{3}, \quad E_{4}=-E_{2}, \\
F_{1}=E_{2}\left(q_{10} q_{6}+q_{7}\right), F_{2}=q_{10} E_{2}, F_{3}=E_{2}\left(q_{10} q_{8}+q_{9}\right), \quad F_{4}=E_{2}\left(q_{1}-q_{10}\right),(40 \mathrm{a}-\mathrm{d}) \\
K_{1}=E_{2}\left(q_{10} q_{2}+q_{3} q_{4}+q_{5}\right), \quad K_{2}=0, \\
M_{1}=E_{2}\left\{\frac{q_{3} D_{\eta 1}}{D_{\xi 2} \mu_{\varphi 2}}\left[d_{1}^{2} \sin \left(d_{1} l_{1}\right)+d_{1} d_{2} \sinh \left(d_{2} l_{1}\right)\right]+\frac{D_{\eta 1}}{D_{\xi 2} \mu_{\varphi 2}}\left[d_{1}^{2} \cos \left(d_{1} l_{1}\right)+d_{2}^{2} \cosh \left(d_{2} l_{1}\right)\right]\right\}, \\
M_{2}=E_{2}\left\{q_{3}\left[-d_{1} \cos \left(d_{1} l_{1}\right)+d_{1} \cosh \left(d_{2} l_{1}\right)\right]+d_{1} \sin \left(d_{1} l_{1}\right)+d_{2} \sinh \left(d_{2} l_{1}\right)\right\} .(42 \mathrm{a}, \mathrm{b})
\end{gathered}
$$

The natural frequencies of the linear modes for out-of-plane motion are given by the roots of the transcendental equation. This arises from boundary condition (33b) using also equations (39-42),

$$
\begin{gathered}
D_{\xi_{1}}\left(q_{10} q_{2}+q_{3} q_{4}+q_{5}\right) \eta_{\varphi} \cos \left(\eta_{\varphi} l_{1}\right)- \\
-\omega_{\text {out }}^{2} I_{\zeta_{2}}\left[q_{3} \sin \left(d_{1} l_{1}\right)+\cos \left(d_{1} l_{1}\right)-\frac{d_{1}}{d_{2}} q_{3} \sinh \left(d_{2} l_{1}\right)-\cosh \left(d_{2} l_{1}\right)\right]+ \\
+I_{\zeta_{2}} \omega_{\text {out }}^{2}\left[\left(q_{10} q_{6}+q_{7}\right) \sin \left(f_{1} l_{2}\right)+q_{10} \cos \left(f_{1} l_{2}\right)+\right. \\
\left.+\left(q_{10} q_{8}+q_{9}\right) \sinh \left(f_{2} l_{2}\right)+\left(q_{1}-q_{10}\right) \cosh \left(f_{2} l_{2}\right)\right]+ \\
+\left(\omega_{\text {out }}^{2} \frac{I_{\zeta_{2}}^{2}}{m_{2}}-D_{\zeta_{2}}\right)\left[\left(q_{1}-q_{10}\right) f_{2}^{2}-q_{10} f_{1}^{2}\right]- \\
-\frac{I_{\zeta_{2}} D_{\zeta_{2}}}{m_{2}}\left[\left(q_{10} q_{6}+q_{7}\right) f_{1}^{4} \sin \left(f_{1} l_{2}\right)+q_{10} f_{1}^{4} \cos \left(f_{1} l_{2}\right)+\right. \\
\left.+\left(q_{10} q_{8}+q_{9}\right) f_{2}^{4} \sinh \left(f_{2} l_{2}\right)+\left(q_{1}-q_{10}\right) f_{2}^{4} \cosh \left(f_{2} l_{2}\right)-q_{10} f_{1}^{2}-\left(q_{1}-q_{10}\right) f_{2}^{2}\right]=0
\end{gathered}
$$

It should be noted that the parameters $q_{i}(i=1 \ldots 10)$ are frequency dependent, and are used to simplify the expressions. They are defined explicitly in Appendix-C. Explicit formulae for the roots of equation (43) are very difficult to obtain. In the case of an L-shaped beam structure with specific dimensions, 
the natural frequencies are the roots of this transcendental equation (43) and can be found easily by examining the zero crossings of the plot of this equation with respect to $\omega_{\text {out }}$.

\subsubsection{Cantilever beam}

In the case of a single cantilever beam in which rotary inertia terms are considered, the equation of motion and boundary conditions arising (e.g. by equation (16a or b), (17), or (19) by eliminating terms which corresponds to the connection with the other beam) are given by,

with boundary conditions,

$$
m \ddot{V}-I \ddot{V}^{\prime \prime}+D V^{I V}=0,
$$

$$
\begin{gathered}
V(0, t)=0, \quad V^{\prime}(0, t)=0, \\
-I \ddot{V}^{\prime}(L, t)+D V^{\prime \prime \prime}(L, t)=0, \quad V^{\prime \prime}(L, t)=0,
\end{gathered}
$$

using the separation of variables by means of,

$$
V(s, t)=Y(s) \cdot a(t),
$$

in equations (44), leads to,

$$
Y^{I V}+\eta^{2}\left(\frac{I}{m}\right) Y^{\prime \prime}-\eta^{2} Y=0, \frac{\ddot{a}(t)}{a(t)}=-\omega_{\text {rot }}^{2}=-\frac{D}{m} \eta^{2},
$$

with boundary conditions,

$$
\begin{array}{cc}
Y(0)=0, & Y^{\prime}(0)=0, \\
\omega_{r o t}^{2} I Y^{\prime}(L)+D Y^{\prime \prime \prime}(L)=0, & Y^{\prime \prime}(L)=0 .
\end{array}
$$

The solution of the equation (47a) with the necessary boundary condition (eq. 48c) and eq. (B.9-B.11), as defined in Appendix-B, leads to a transcendental equation. The roots of this transcendental equation define the natural frequencies of the beam in bending, considering also the inertia terms. This transcendental equation is given by,

$$
\begin{aligned}
& D\left[-\sigma_{1, S}^{3} \cos \left(\sigma_{1} L\right)-D_{L} \sigma_{1, S}^{3} \sin \left(\sigma_{1} L\right)-\sigma_{1, S} \sigma_{2, S}^{2} \cosh \left(\sigma_{2, S} L\right)+D_{L} \sigma_{2, S}^{3} \sinh \left(\sigma_{2, S} L\right)\right]+ \\
& +\omega_{\text {rot }}^{2} I\left[\sigma_{1, S} \cos \left(\sigma_{1, S} L\right)+D_{L} \sigma_{1, S} \sin \left(\sigma_{1, S} L\right)-\sigma_{1, S} \cosh \left(\sigma_{2, S} L\right)+D_{L} \sigma_{2, S} \sinh \left(\sigma_{2, S} L\right)\right]=0,
\end{aligned}
$$

In the case where rotary inertia terms are neglected the transcendental equation (49) when eq. $(47 \mathrm{~b}, \mathrm{~B} .12)$ is considered, takes the form,

$$
\begin{gathered}
-\cos \left(\sqrt[4]{\left(m \cdot \omega_{0}^{2}\right) / D} \cdot L\right)-D_{L, 0} \sin \left(\sqrt[4]{\left(m \cdot \omega_{0}^{2}\right) / D} \cdot L\right)-\cosh \left(\sqrt[4]{\left(m \cdot \omega_{0}^{2}\right) / D} \cdot L\right)+ \\
+D_{L, 0} \sinh \left(\sqrt[4]{\left(m \cdot \omega_{0}^{2}\right) / D} \cdot L\right)=0,
\end{gathered}
$$


for which we also have,

$$
D_{L, 0}=\frac{\sin \left(\sqrt[4]{\left(m \cdot \omega_{0}^{2}\right) / D^{\cdot L}}\right)+\sinh \left(\sqrt[4]{\left(m \cdot \omega_{0}^{2}\right) / D^{\cdot L}}\right)}{\cos \left(\sqrt[4]{\left(m \cdot \omega_{0}^{2}\right) / D^{\prime}} \cdot L\right)+\cosh \left(\sqrt[4]{\left(m \cdot \omega_{0}^{2}\right) / D^{\cdot L}}\right)} .
$$

The roots of equation (50) with respect to $\omega_{0}$ denote the natural frequencies of a cantilever beam, when the rotary inertia terms are neglected.

\section{Models}

In order to validate the theoretical results of modal analysis, four FE models were constructed in the Abaqus program consisting of two sets of beams (with indices $a, b$ respectively). The material for all the models was aluminium of density, $\rho=2800 \mathrm{~kg} / \mathrm{m}^{3}$, Young's modulus $E=70 \mathrm{MPa}$, Poisson's ratio $v=0.33$, and shear modulus, $\mathrm{G}_{12}=26.32 \mathrm{MPa}$. The dimensions of the beams for each set are,

$$
\begin{gathered}
l_{1 a} \times b_{1 a} \times h_{1 a}=0.2 \mathrm{~m} \times 0.004 \mathrm{~m} \times 0.003 \mathrm{~m}, \\
l_{2 a} \times b_{2 a} \times h_{2 a}=0.15 \mathrm{~m} \times 0.004 \mathrm{~m} \times 0.003 \mathrm{~m}, \\
l_{1 b} \times b_{1 b} \times h_{1 b}=0.18 \mathrm{~m} \times 0.01295 \mathrm{~m} \times 0.00216 \mathrm{~m}, \\
l_{2 b} \times b_{2 b} \times h_{2 b}=0.21 \mathrm{~m} \times 0.01295 \mathrm{~m} \times 0.00216 \mathrm{~m} .
\end{gathered}
$$

For each set of dimensions two different models were constructed with two different orientations of the secondary beam (Figures 2a,c). In the first orientation the secondary beam is oriented in such a way that its width is in the same direction as the width of the primary beam (Figure 2a). In the second model the secondary beam has a transversal orientation (Figure 2c).

The parameters used in equations $(14,16)$ are defined as follows:

a) For model 1 with dimensions given for the first set of beams with the first orientation of the secondary beam, and also considering the warping coefficient for torsional rigidity [6],

$$
\begin{gathered}
m_{1, a}=\rho b_{1 a} h_{1 a}=0.034 \mathrm{~kg} / \mathrm{m}, \quad I_{\eta_{1, a}}=\rho \frac{h_{1 a} \cdot b_{1 a}^{3}}{12}=4.480 \times 10^{-8} \mathrm{~kg} \mathrm{~m}, \\
I_{\xi_{1, a}}=\rho\left(\frac{h_{1 a} \cdot b_{1 a}^{3}+b_{1 a} \cdot h_{1 a}^{3}}{12}\right)=7.000 \times 10^{-8} \mathrm{~kg} \mathrm{~m}, \quad D_{\zeta_{1, a}}=E \frac{b_{1 a} \cdot h_{1 a}^{3}}{12}=0.630 \mathrm{~Pa} \mathrm{~m}^{4}, \\
D_{\eta_{1, a}}=E \frac{h_{1 a} \cdot b_{1 a}^{3}}{12}=1.120 \mathrm{~Pa} \mathrm{~m}^{4}, \\
D_{\xi_{1, a}}=G_{12} \frac{1}{3} b_{1 a} h_{1 a}^{3}\left(1-\frac{192 h_{1 a}}{\pi^{5} b_{1 a}} \sum_{n=1,3, \ldots}^{\infty}\left(\frac{1}{n^{5}} \tanh \left(\frac{n \pi b_{1 a}}{2 h_{1 a}}\right)\right)\right)=0.513 \mathrm{~Pa} \mathrm{~m}^{4}, \\
m_{2, a}=\rho b_{2 a} h_{2 a}=0.034 \mathrm{~kg} / \mathrm{m}, \quad I_{\zeta_{2, a}}=\rho \frac{h_{2 a} \cdot b_{2 a}^{3}}{12}=4.480 \times 10^{-8} \mathrm{~kg} \mathrm{~m}, \\
I_{\xi_{2, a}}=\rho\left(\frac{h_{2 a} \cdot b_{2 a}^{3}+b_{2 a} \cdot h_{2 a}^{3}}{12}\right)=7.000 \times 10^{-8} \mathrm{~kg} \mathrm{~m}, \quad D_{\zeta_{2, a}}=E \frac{h_{2 a} \cdot b_{2 a}^{3}}{12}=1.120 \mathrm{~Pa} \mathrm{~m}^{4}, \\
D_{\eta_{2, a}}=E \frac{b_{2 a} \cdot h_{2 a}^{3}}{12}=0.630 \mathrm{~Pa} \mathrm{~m}^{4}, \\
D_{\xi_{2, a}}=G_{12} \frac{1}{3} b_{2 a} h_{2 a}^{3}\left(1-\frac{192 h_{2 a}}{\pi^{5} b_{2 a}} \sum_{n=1,3, \ldots}^{\infty}\left(\frac{1}{n^{5}} \tanh \left(\frac{n \pi b_{2 a}}{2 h_{2 a}}\right)\right)\right)=0.513 \mathrm{~Pa} \mathrm{~m}^{4},
\end{gathered}
$$

b) For model 2 and using the first set of dimensions but with the transversal orientation of the secondary beam,

$$
\begin{gathered}
I_{\zeta_{2, a, t}}=\rho \frac{b_{2 a} \cdot h_{2 a}^{3}}{12}=2.520 \times 10^{-8} \mathrm{~kg} \mathrm{~m}, \quad D_{\zeta_{2, a, t}}=E \frac{b_{2 a} \cdot h_{2 a}^{3}}{12}=0.630 \mathrm{~Pa} \mathrm{~m}^{4}, \\
D_{\eta_{2, a, t}}=E \frac{h_{2 a} \cdot b_{2 a}^{3}}{12}=1.120 \mathrm{~Pa} \mathrm{~m}^{4},
\end{gathered}
$$


the other parameters are the same as those for the first orientation of the secondary beam (model 1).

c) For model 3 the dimensions are given by the second set of beams and they take the first orientation of the secondary beam, being defined by,

$$
\begin{gathered}
m_{1, b}=\rho b_{1 b} h_{1 b}=0.078 \mathrm{~kg} / \mathrm{m}, \quad I_{\eta_{1 b}}=\rho \frac{h_{1 b} \cdot b_{1 b}^{3}}{12}=1.095 \times 10^{-6} \mathrm{~kg} \mathrm{~m}, \\
I_{\xi_{1, b}}=\rho\left(\frac{h_{1 b} \cdot b_{1 b}^{3}+b_{1 b} \cdot h_{1 b}^{3}}{12}\right)=1.125 \times 10^{-6} \mathrm{~kg} \mathrm{~m}, \quad D_{\zeta_{1, b}}=E \frac{b_{1 b} \cdot h_{1 b}^{3}}{12}=0.761 \mathrm{~Pa} \mathrm{~m}^{4}, \\
D_{\eta_{1, b}}=E \frac{h_{1 b} \cdot b_{1 b}^{3}}{12}=27.364 \mathrm{~Pa} \mathrm{~m}^{4} \\
D_{\xi_{1, b}}=G_{12} \frac{1}{3} b_{1 b} h_{1 b}^{3}\left(1-\frac{192 h_{1 b}}{\pi^{5} b_{1 b}} \sum_{n=1,3, \ldots}^{\infty}\left(\frac{1}{n^{5}} \tanh \left(\frac{n \pi b_{1 b}}{2 h_{1 b}}\right)\right)\right)=1.024 \mathrm{~Pa} \mathrm{~m}^{4}, \\
m_{2, b}=\rho b_{2 b} h_{2 b}=0.078 \mathrm{~kg} / \mathrm{m}, \quad I_{\zeta_{2, b}}=\rho \frac{h_{2 b} \cdot b_{2 b}^{3}}{12}=1.095 \times 10^{-6} \mathrm{~kg} \mathrm{~m}, \\
I_{\xi_{2, b}}=\rho\left(\frac{h_{2 b} \cdot b_{2 b}^{3}+b_{2 b} \cdot h_{2 b}^{3}}{12}\right)=1.125 \times 10^{-6} \mathrm{~kg} \mathrm{~m}, \quad D_{\zeta_{2, b}}=E \frac{h_{2 b} \cdot b_{2 b}^{3}}{12}=27.364 \mathrm{~Pa} \mathrm{~m}^{4}, \\
D_{\eta_{2, b}}=E \frac{b_{2 b} \cdot h_{2 b}^{3}}{12}=0.761 \mathrm{~Pa} \mathrm{~m}^{4}, \\
D_{\xi_{2, b}}=G_{12} \frac{1}{3} b_{2 b} h_{2 b}^{3}\left(1-\frac{192 h_{2 b}}{\pi^{5} b_{2 b}} \sum_{n=1,3, \ldots}^{\infty}\left(\frac{1}{n^{5}} \tanh \left(\frac{n \pi b_{2 b}}{2 h_{2 b}}\right)\right)\right)=1.024 \mathrm{~Pa} \mathrm{~m}^{4},
\end{gathered}
$$

d) Finally for model 4, using the second set of dimensions but with the secondary beam oriented in the transversal manner, they are given by,

$$
\begin{gathered}
I_{\zeta_{2, a, t}}=\rho \frac{b_{2 a} \cdot h_{2 a}^{3}}{12}=3.045 \times 10^{-8} \mathrm{~kg} \mathrm{~m}, \quad D_{\zeta_{2, a, t}}=E \frac{b_{2 a} \cdot h_{2 a}^{3}}{12}=0.761 \mathrm{~Pa} \mathrm{~m}^{4}, \\
D_{\eta_{2, a, t}}=E \frac{h_{2 a} \cdot b_{2 a}^{3}}{12}=27.364 \mathrm{~Pa} \mathrm{~m}^{4},
\end{gathered}
$$

The other parameters are the same as those for the first orientation of the secondary beam (model 3).

According to Vlasov's theory the shear effects are dominant only in cases where the dimensions with respect to the length have a ratio higher than 0.1 [9], and for the second set of dimensions (models 3 and 4) we expect to observe some shear effects for the higher modes. This is due to the fact that the widths in the second set of beams are one order of magnitude higher than those of the first set of beams, whereas the lengths are of the same order.

Before modelling the L-shaped beams a modal analysis of a single cantilever beam was performed in Abaqus, with the dimensions used for the L-shaped beam structure. We modelled them using two kinds of elements, denoted as wire (B31) and shell (S4) elements, and also considering shear effects. The difference in the results obtained by using these two kinds of elements, with examination restricted to the first 5 bending modes for each one motion (in-plane and out-of-plane bending), was less than $1 \%$. Therefore only the results of the model with wire elements are presented here noting that 150 elements were used for all cases. In order to perform a comparison between the mode shapes we selected specific points on each beam which corresponded to a division of the beam length into 30 elements for all cases.

Furthermore the four FE models of the L-shaped beam structures were constructed in Abaqus in order to perform numerical modal analysis. For each model, and to ensure correct numerical results, two kinds of elements were used in the form of wire (B31) and shell (S4) elements, for which shear effects were also considered. In the case of wire modelling, 150 elements were used for each beam. In the case of shell modelling and a) model-1, 150 elements in the longitudinal direction $\times 30$ elements for each beam, were used and b) models- 2 and 4, 151 elements in the longitudinal direction $\times 16$ elements for the primary beam and 150 elements in the longitudinal direction $\times 16$ elements for the secondary beam, were used and c) in model-3, 150 elements in the longitudinal direction $\times 16$ elements for all beams were used. In order to compare mode shapes between the theory and the FE simulations we selected specific points in each beam. In the case of models 1 and 3, the selection of points corresponds to division of the beam lengths into 30 elements. In the case of models 2 and 4 the 
primary beam was divided into 31 elements and the secondary beam was divided into 30 elements.

\section{Results-Discussion}

In order to validate the theoretical modal analysis the theoretical results were compared with the FE simulations in terms of the natural frequencies and mode shapes. The comparison of mode shapes was carried out using the Modal Assurance Criterion (MAC). The MAC comparison between two mode shape vectors $\mathrm{Y}_{\mathrm{MAC}, 1}$ and $\mathrm{Y}_{\mathrm{MAC}, 2}$ can be estimated using the following formula [8],

$$
\operatorname{MAC}\left(Y_{M A C, 1}, Y_{M A C, 2}\right)=\frac{\left|\left\{Y_{M A C, 1}\right\}^{T}\left\{Y_{M A C, 2}\right\}\right|^{2}}{\left(\left\{Y_{M A C, 1}\right\}^{T}\left\{Y_{M A C, 1}\right\}\right)\left(\left\{Y_{M A C, 2}\right\}^{T}\left\{Y_{M A C, 2}\right\}\right)} .
$$

noting that the superscript $\mathrm{T}$ defines the transpose.

In the case of in-plane motion of the L-shaped beam structure the mode shape is given by,

$$
\left\{Y_{M A C}\right\}=\left[\left\{Y_{v}\right\},\left\{Y_{W}\right\}\right]^{T},
$$

for which the mode shape vector is determined at the aforementioned selected points. In the case of out-of-plane motion for the structure then the mode shape is given by,

$$
\left\{Y_{M A C}\right\}=\left[\left\{Y_{V}\right\},\left\{Y_{w}\right\},\left\{Y_{\Phi}\right\},\left\{Y_{\varphi}\right\}\right]^{T}
$$

Figures 2a,b (c,d) depict the mode shapes using Abaqus for the 10th modes of in-plane and out-ofplane motion, respectively, for model 1 (model 2).

\subsection{Results for single beams}

Theoretical modal analysis was performed for a single cantilever beam in bending by finding the roots of transcendental equations (49) considering the inertia terms and (50) neglecting the inertia terms for all the beams used in the modelling of the L-shaped structure. The dimensions are defined in the previous section and the inertia and stiffness terms are defined by equations (53-56). For both sets of beam dimensions the results for the primary and the secondary beam are similar, therefore only an analysis for the secondary beam is presented in this paper. In Tables 1.1-2 (1.3-4) the results of the examination of the modal analysis of the secondary beam of model 1 (model 2) in bending are shown. The frequencies which resulted from theoretical analysis, with and without considering the inertia terms, are presented there, and also the frequencies from the FE simulations. There is also a comparison of the natural frequencies from the theoretical results which demonstrates the effect of the rotary inertia terms, and a comparison of the theoretical with the FE simulation results in terms of frequencies and mode shapes. In the case of the in-plane bending of model 1 (Table 1.1) the inertia terms clearly have no significant effect. The comparison of theoretical results with FE simulations, shown in Table 1.1, shows that they are in very good agreement in terms of frequencies and MAC. Therefore it may be concluded that the shear effect for the first five modes is not significant. In the case of out-of-plane bending of the secondary beam of model 1, the results are shown in Table 1.2.The inertia and shear effects are also insignificant there. The results for the secondary beam of model 2 are shown in Tables 1.3 (1.4) for in-plane bending (out-of-plane bending). In the case of in-plane bending the shear and rotary inertia effects are insignificant but in out-of-plane bending of the secondary beam (model 2) the shear and rotary inertia effects are more dominant, as shown in Table 1.4. 


\subsection{In-plane motions of L-shaped beam structure}

Considering the in-plane motions in this structure then a comparison of the theoretical and numerical results for the four models, taking into account just the first 10 modes, is presented in Tables 1.5-1.7. In Table 1.5 the theoretical results for model 1 are in a very good agreement with those from FE simulations. There is no significant difference between the results of the wire and the shell element models, and the maximum relative difference between the numerical and theoretical results is $2 \%$, whereas the MAC diagonal values are 1.00. Figure 3a depicts the theoretical and numerical (wire model) results for the $10^{\text {th }}$ mode shape for both beams (the same scale coefficient is used for both beams undergoing theoretical mode shapes) with a good agreement and this confirms the high MAC values.

In the case of model 2 for which the secondary beam is placed in the transversal configuration, the results are shown in Table 1.6. In this table there is a good agreement between the natural frequencies for both models, with less than a $4 \%$ difference, for the first nine modes. Therefore, the shear effect influence is minimal. In the case of the wire model for the $1^{\text {st }}$ mode and then in the case of the shell model for the $9^{\text {th }}$ mode there is a low MAC value due to numerical errors. It should be highlighted that in the $10^{\text {th }}$ mode there is greater discrepancy in the natural frequencies for both models, and also there are low MAC values due to the imposed inextensionality conditions. More precisely, the axial displacement of the primary beam due to the inextensionality condition, is a second order nonlinear term, and this has been neglected from the formulation. Figure $3 \mathrm{~b}$ depicts the theoretical and numerical wire model $10^{\text {th }}$ mode shape for the $2^{\text {nd }}$ model, using the same scale coefficient for both beams for the theoretical mode shape. Examining the FE mode shape of the secondary beam in Figure $3 \mathrm{~b}$ it is possible to notice a displacement at the clamped end $(\mathrm{x}=0)$ which is due to the axial displacement of the primary beam, this was neglected in the theoretical modal analysis. Therefore the coupling between bending in the secondary beam and the axial motion of the primary beam has been neglected, and so this axial displacement makes a significant difference to this mode shape. The mode shape from the shell model in Abaqus is presented in Figure 2b, in which the axial displacement or shortening of the primary beam is visible. The theoretical and numerical results for model 3, presented in Table 1.7, are in a good agreement, with a minimal shear effect. This is justified further on from examination of Figure 3c. In this figure (Fig.3c), the theoretical and numerical results for the wire model of the $10^{\text {th }}$ mode shape are depicted with the same scale coefficient for both beams in the theoretical mode shapes, and they are in a good agreement.

In model 4 the secondary beam is oriented in the transversal direction, therefore in the higher modes it is expected that the influence of shear effects will be apparent. The results of modal analysis for model 4 are shown in Table 1.8, in which the theoretical results are in a relatively good agreement with the finite element simulations results. The differences in the natural frequencies of the theoretical results with those of the corresponding finite element simulations are higher than the differences shown by the other models e.g. Tables 1.5-7. Detailed examination of the mode shapes for this model (4) indicates that the low MAC values for the $6^{\text {th }}$ and $9^{\text {th }}$ modes of the B31 wire element model in Table 1.8 are due to numerical errors. Similarly, the low MAC values for the $5^{\text {th }}$ and $8^{\text {th }}$ modes in the case of the S4 shell element model are also due to numerical errors. For both the B31 and S4 element models the low MAC values in Table 1.8 for the $7^{\text {th }}$ mode seem to be due to shear effects. Examination of the $10^{\text {th }}$ mode shapes of the finite element models shows, apart from the influence of some shear effects, that there is also significant axial displacement in the primary beam, and this is similarly for model 2 , and it explains the low MAC values and the relative differences in the natural frequencies (Table 1.8) for this mode. Figure $3 \mathrm{~d}$ depicts the theoretical and numerical results for the wire element model specifically the $10^{\text {th }}$ mode. By considering the mode shape of the secondary beam from the finite element simulation in Figure $3 \mathrm{~d}$, it is clear that at the clamped end $(x=0)$ there is a significant displacement caused by the axial displacement of the primary beam, and this explains the low MAC values for this mode. Also it is expected that shear effects will be more dominant in this mode due to significant displacements of the secondary beam.

In conclusion, for in-plane motion the comparison of theoretical with numerical results shows a relatively good mutual agreement, which tends to validate the analytical work undertaken for in-plane motion. 


\subsection{Out-of-plane motions of the L-shaped beam}

The theoretical and FE simulations results for all the models and the comparison considering the outof-plane motions are all shown in Tables 1.9-1.12. Similarly to the case of in-plane motion, these Tables show the theoretical and numerical wire and shell model natural frequencies, the $\%$ relative difference between the theoretical and numerical results and also the MAC analysis for the comparison of the theoretical mode shapes with each one of the mode shapes from the FE simulations. Table 1.9 presents the results for model 1 . Both FE models and theory are in a very good agreement with $1 \%$ of maximum relative difference in the natural frequencies, and the MAC diagonal is 1.00 , all across the modes. Figures $4 \mathrm{a}, \mathrm{b}$ depict the theoretical and FE wire model simulation mode shape for the $10^{\text {th }}$ mode of the $1^{\text {st }}$ model, noting that all the theoretical motions for bending and torsion have the same scale coefficient. More precisely, Figure $4 \mathrm{a}(\mathrm{b})$ depicts the mode shape in bending (torsion), and there is a good agreement between the theoretical and FE simulations which confirms the MAC value. The results for model 2 in Table 1.10 present again a good agreement between the FE simulations with the theoretical values. The maximum relative difference is $2 \%$ in the natural frequencies, and the MAC diagonal is equal to 1.00 for all the mode shapes. Figures $4 \mathrm{c}$,d depict the theoretical and FE wire model simulations for mode 10 for the $2^{\text {nd }}$ model. In Figure $4 \mathrm{c}(\mathrm{d})$ the theoretical and FE simulation mode shape in bending (torsion) are in a good agreement, which validates the high MAC value. Table 1.11 shows the results for model 3, in which the theory is seen to be in good accordance with the FE simulations. The highest relative difference in natural frequencies is $4 \%$ for the $9^{\text {th }}$ mode noting that there is clear evidence of shear effect influences, and that all the others have a maximum of $3 \%$ difference. For all the mode shapes the MAC diagonals show high values, apart from mode 9 which is just acceptable. Figures 5a, b depict the theoretical and FE wire model mode shapes for mode 10 for model No.3. It should be noted that in bending there is some difference in the mode shapes (Fig.5a) but the displacement values are very small, due to numerical errors. The dominant motions in this mode are the rotations (Fig. 5b). There is a very good agreement between the theoretical and numerical simulation mode shapes (Fig.5a b), which is confirmed by the MAC value in Table 1.11. The results for model 4 show a very good agreement between the theory and the FE simulation results as shown in Table 1.12. The maximum relative difference in the natural frequencies is $1 \%$ and the MAC values are between $0.99-1.00$, neglecting the $7^{\text {th }}$ mode for the wire elements model which is again lower due to numerical error. Figures $5 \mathrm{c}, \mathrm{d}$ depict the theoretical and FE wire model simulation mode shape for mode 10 for the $4^{\text {th }}$ model. Also in Figure $5 \mathrm{c}(\mathrm{d})$ the mode shapes in bending (torsion) suggest very good agreement between the theoretical and the FE simulations, and this justifies the high MAC value in Table 1.12.

In conclusion, there is very a good agreement in the natural frequencies with a maximum relative difference of $4 \%$ only in one case, and between 0.99-1.00 MAC values, apart from one case which is due to shear effect influence. These results validate the theoretical analysis for out-of-plane motion, and also the comparison shows that shear effect influences are negligible for the modes examined.

\section{Conclusions}

In this article we performed a theoretical modal analysis of an L-shaped beam structure, by solving two sets of coupled equations of motion for in-plane bending and out-of-plane bending and torsion. Two sets of dimensions were considered for the FE models. For each beam a theoretical and numerical modal analysis was performed to examine the inertia and shear effects. It was shown for both models that for in-plane bending the shear and inertia effects are insignificant and for out-ofplane bending the shear and inertia effects are more significant especially for the second beam.

Then, four models of the L-shaped beam structure were constructed with two configurations of the secondary beam and the second one of these was oriented in the transversal direction. Both theoretical and numerical modal analyses were performed for the four models, and the results were compared. For both solutions, for in-plane motion and out-of-plane motion there is a good agreement between the theory and the FE simulations, which tends to validate the theoretical analysis. In the case of in-plane motion for the two models, in the $10^{\text {th }}$ mode there is a high discrepancy due to the coupling 
of axial motion of the primary beam with the in-plane bending motion of the secondary beam, which was neglected in the theoretical approach. Also in some of the higher modes some discrepancies occurred due to shear effects. Considering the out-of-plane motions, a comparison of the theoretical with numerical results shows smaller differences and in these cases the shear effects are negligible. It should be noted that although in the case of a simple cantilever beam the shear effect is more dominant even in the lower modes, in the L-shaped beam structure the situation is different. In inplane motion of the L-shaped beam the first mode of each beam is coupled together, which results in 2 modes. Therefore, a shifting of the corresponding second mode of the cantilever beam to a higher mode in the L-shaped beam structure is expected. Considering this phenomenon the shear effects attributed, generally speaking, to higher modes are shifted to even higher modes in the case of the Lshaped beam structure.

Finally, the theoretical modal analysis performed in this article for an L-shaped beam structure can give relatively accurate results for the first modes, and will be used to discretise the nonlinear equations for the second order approximation, with a projection to the linear mode shapes in order to continue with nonlinear modal analysis of the L-shaped beam structure.

\section{Acknowledgement}

The research leading to these results has received funding from the European Union Seventh Framework Programme (FP7/2007-2013), FP7 - REGPOT - 2009 - 1, under grant agreement No:245479. The support by the Polish Ministry of Science and Higher Education - Grant No 1471-1/7.PR UE/2010/7 is also acknowledged by the second author.

\section{References}

1. Roberts, J.W., Cartmell, M.P., 1984, Forced vibration of a beam system with autoparametric coupling effects, Strain, 20, 123-131.

2. Cartmell, M.P., Roberts, J.W., 1987, Simultaneous combination resonances in a parametrically excited cantilever beam, Strain, 23, 117-126.

3. Balachandran, B., Nayfeh A.H., 1990, Nonlinear motion of beam-mass structure, Nonlinear Dynamics, 1, 39-61.

4. Warminski, J., Cartmell, M.P., Bochenski, M., Ivanov, I., 2008, Analytical and experimental investigations of an autoparametric beam structure, Journal of Sound and Vibration, 315, 486-508. 5. Ozonato, N., Nagai, K.-I., Maruyama, S., Yamaguchi, T., 2012, Chaotic Vibrations of A PostBuckled L-Shape Beam with an Axial Constraint, Nonlinear Dynamics, 67, 2363-2379.

6. Nayfeh, A.H., Pai, F., 2004, Linear and Nonlinear Structural Mechanics, first edition, John Willey and sons, New Jersey.

7. Georgiades, F., Warminski, J., Cartmell, P., M., Towards Linear Modal Analysis for an L-shaped Beam: Equations of Motion, Mechanics Research Communications, under review.

8. Ewins, D.J., 2000, Modal Testing (Theory, Practice and Application), second edition, Research Studies Press Ltd.

9. Librescu, L., Song, O., 2006, Thin-Walled Composite Beams (Theory and Application), Springer.

\section{Appendix-A}

In this appendix the coefficients of the mode shapes (eq. 24a,b) are determined for in-plane bending, through the boundary conditions (eq. 23a-c,e-h). All the coefficients are stated as functions of constant $\mathrm{C}_{1}$.

Starting from equation 23a and using eq. 24a, we get,

$$
\mathrm{C}_{4}=-\mathrm{C}_{2} \text {, }
$$


also, by using equation $23 \mathrm{~b}$, and then considering eq. $24 \mathrm{a}$, it is shown that,

$$
\mathrm{C}_{3}=-\mathrm{C}_{1},
$$

Applying equation $23 \mathrm{c}$ with equation $24 \mathrm{a}$ and then taking equations $25 \mathrm{a}$, b, we get,

$$
\mathrm{C}_{2}=\mathrm{C}_{1}\left\{\frac{-\cos \left(\mu_{v} l_{1}\right)-\cosh \left(\mu_{v} l_{1}\right)+\frac{m_{2} l_{2} \mu_{v}}{m_{1}}\left[\sin \left(\mu_{v} l_{1}\right)-\sinh \left(\mu_{v} l_{1}\right)\right]}{\sinh \left(\mu_{v} l_{1}\right)-\sin \left(\mu_{v} l_{1}\right)+\frac{m_{2} l_{2} \mu_{v}}{m_{1}}\left[\cosh \left(\mu_{v} l_{1}\right)-\cos \left(\mu_{v} l_{1}\right)\right]}\right\}=\mathrm{C}_{1} \mathrm{C}_{L}
$$

Therefore the constants in equation $24 \mathrm{a}$ are given by equations $25 \mathrm{a}, \mathrm{b}$ and also by,

Equation $23 \mathrm{e}$ with equation $24 \mathrm{~b}$ leads to,

$$
\mathrm{C}_{4}=-\mathrm{C}_{1}\left\{\frac{-\cos \left(\mu_{v} l_{1}\right)-\cosh \left(\mu_{v} l_{1}\right)+\frac{m_{2} l_{2} \mu_{v}}{m_{1}}\left[\sin \left(\mu_{v} l_{1}\right)-\sinh \left(\mu_{v} l_{1}\right)\right]}{\sinh \left(\mu_{v} l_{1}\right)-\sin \left(\mu_{v} l_{1}\right)+\frac{m_{2} l_{2} \mu_{v}}{m_{1}}\left[\cosh \left(\mu_{v} l_{1}\right)-\cos \left(\mu_{v} l_{1}\right)\right]}\right\}=-\mathrm{C}_{1} \mathrm{C}_{L} .
$$

$$
\mathrm{D}_{4}=-\mathrm{D}_{2},
$$

then making use of equation 23g, and equation 24b, and then taking into account (A5), we have,

$$
\mathrm{D}_{3}=D_{1}\left(\frac{\sin \left(\mu_{W} l_{2}\right)}{\sinh \left(\mu_{W} l_{2}\right)}\right)+D_{2}\left(\frac{\cos \left(\mu_{W} l_{2}\right)+\cosh \left(\mu_{W} l_{2}\right)}{\sinh \left(\mu_{W} l_{2}\right)}\right)
$$

Equation 23h, and equation 24b, A.5-6, leads to,

$$
D_{2}=D_{1}\left\{\frac{\cos \left(\mu_{W} l_{2}\right) \sinh \left(\mu_{W} l_{2}\right)-\sin \left(\mu_{W} l_{2}\right) \cosh \left(\mu_{W} l_{2}\right)}{\sin \left(\mu_{W} l_{2}\right) \sinh \left(\mu_{W} l_{2}\right)+\cos \left(\mu_{W} l_{2}\right) \cosh \left(\mu_{W} l_{2}\right)+1}\right\}
$$

Therefore, using A.7 in A.6 we obtain,

$\mathrm{D}_{3}=D_{1}\left\{\frac{\sin \left(\mu_{W} l_{2}\right)}{\sinh \left(\mu_{W} l_{2}\right)}+\frac{\left[\cos \left(\mu_{W} l_{2}\right) \sinh \left(\mu_{W} l_{2}\right)-\sin \left(\mu_{W} l_{2}\right) \cosh \left(\mu_{W} l_{2}\right)\right]\left[\cos \left(\mu_{W} l_{2}\right)+\cosh \left(\mu_{W} l_{2}\right)\right]}{\sinh \left(\mu_{W} l_{2}\right)\left[\sin \left(\mu_{W} l_{2}\right) \sinh \left(\mu_{W} l_{2}\right)+\cos \left(\mu_{W} l_{2}\right) \cosh \left(\mu_{W} l_{2}\right)+1\right]}\right\}=D_{1} C_{g}$.

Finally, using 23f, with 24, 25a,b, A.5,7,8, gives the following,

or,

$$
D_{1}=-\frac{\mu_{v}\left\{\cos \left(\mu_{v} l_{1}\right)-\cosh \left(\mu_{v} l_{1}\right)+C_{L}\left[-\sin \left(\mu_{v} l_{1}\right)-\sinh \left(\mu_{v} l_{1}\right)\right]\right\}}{\mu_{W}\left(1+C_{g}\right)} C_{1},
$$

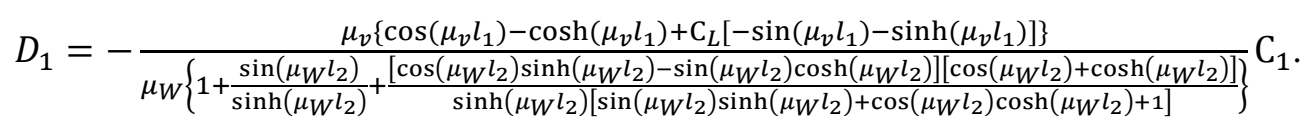

\section{Appendix-B}

The general solution of the ODE which arises from the out-of-plane bending and takes the inertia terms into account is not trivial and is presented within this appendix.

In both cases of out-of-plane bending the ODEs (eq. 31a,b) are structurally the same but with different coefficients, and in general can be written as follows,

$$
Y^{I V}+b^{2} d Y^{\prime \prime}-b^{2} Y=0 .
$$

The characteristic polynomial for this form of ODE is,

with $\mathrm{d}>0$.

$$
P(k)=k^{4}+b^{2} d k^{2}-b^{2}=0,
$$

This equation is trivial to solve since it is a bi-squared polynomial, therefore the roots are given by, 


$$
\begin{gathered}
k_{1,2}= \pm \sqrt{-\frac{\left(b^{2} d+\sqrt{b^{4} d^{2}+4 b^{2}}\right)}{2}}= \pm i \sqrt{\frac{b^{2} d+\sqrt{b^{4} d^{2}+4 b^{2}}}{2}}= \pm i \sigma_{1}, \quad \text { (imaginary), } \\
k_{3,4}= \pm \sqrt{\frac{-b^{2} d+\sqrt{b^{4} d^{2}+4 b^{2}}}{2}}= \pm \sqrt{\frac{-b^{2} d+\sqrt{b^{4} d^{2}+4 b^{2}}}{2}}= \pm \sigma_{2}, \quad \text { (real). (B.3) }
\end{gathered}
$$

Considering the roots (B.3) of the characteristic polynomial (B.2) the general solution of equation (B.1) is given by,

$$
Y(s)=B_{1} \exp \left(i \sigma_{1} s\right)+B_{2} \exp \left(-i \sigma_{1} s\right)+B_{3} \exp \left(\sigma_{2} s\right)+B_{4} \exp \left(-\sigma_{2} s\right)
$$

Using the hyperbolic and trigonometric identities,

we have,

$$
\begin{array}{r}
\sinh (x)=\frac{\exp (x)-\exp (-x)}{2}, \quad \cosh (x)=\frac{\exp (x)+\exp (-x)}{2}, \\
\sin (x)=\frac{\exp (i x)-\exp (-i x)}{2 i}, \quad \cos (x)=\frac{\exp (i x)+\exp (-i x)}{2},
\end{array}
$$

$$
\begin{aligned}
& \exp (x)=\sinh (x)+\cosh (x), \exp (-x)=\cosh (x)-\sinh (x), \\
& \exp (i x)=i \sin (x)+\cos (x), \exp (-i x)=\cos (x)-i \sin (x)
\end{aligned}
$$

Therefore substituting relations (B.6a-d) in equation (B.4), and after some algebraic manipulations, we get the final form of the general solution of equation (B.1),

$$
Y(s)=L_{1} \sin \left(\sigma_{1} s\right)+L_{2} \cos \left(\sigma_{1} s\right)+L_{3} \sinh \left(\sigma_{2} s\right)+L_{4} \cosh \left(\sigma_{2} s\right),
$$

where the constants $\mathrm{L}_{\mathrm{i}}(\mathrm{i}=1, . .4)$ are determined through the boundary conditions.

Equation $47 \mathrm{a}$ is in the form of eq. (B.1) therefore the mode shapes are as given by equation (B.7), with,

$$
\begin{aligned}
& \sigma_{1, S}=\sqrt{\frac{\eta^{2}\left(\frac{I}{m}\right)+\sqrt{\eta^{4}\left(\frac{I}{m}\right)^{2}+4 \eta^{2}}}{2}}, \\
& \sigma_{2, S}=\sqrt{\frac{-\eta^{2}\left(\frac{I}{m}\right)+\sqrt{\eta^{4}\left(\frac{I}{m}\right)^{2}+4 \eta^{2}}}{2}} .
\end{aligned}
$$

Considering equation (48a) and eq. (B.7) we get,

$$
L_{4}=-L_{2},
$$

Also, equation (48b) and eq. (B.7,9), give,

$$
L_{3}=-\frac{\sigma_{1, s}}{\sigma_{2, s}} L_{1},
$$

Then, taking equation (48d), and eq. ( B.7,9-10), yields,

$$
L_{2}=-L_{1}\left[\frac{\sigma_{1, s}^{2} \sin \left(\sigma_{1, s} L\right)+\sigma_{1, s} \sigma_{2, s} \sinh \left(\sigma_{2, S} L\right)}{\sigma_{1, s}^{2} \cos \left(\sigma_{1, S} L\right)+\sigma_{2, s}^{2} \cosh \left(\sigma_{2, S} L\right)}\right]=-L_{1} D_{L} .
$$


In the case when the inertia terms are neglected (I=0) equation (B.3) takes the form,

$$
\sigma_{1,0}=\sigma_{2,0}=\sqrt{\eta}
$$

\section{Appendix-C}

In this appendix, the coefficients of the mode shapes for out-of-plane motion are obtained (eq. 36,38).

Starting from equation (31a), and using equation (36a), we get,

$$
K_{2}=0 \text {. }
$$

Also, taking equation (32a), and eq. (36a), gives,

$$
E_{4}=-E_{2}
$$

Then applying equation (32b), and eq. (36a, C.2), shows that,

$$
E_{3}=-\left(d_{1} / d_{2}\right) E_{1}
$$

Equation (34a), and eq. (36a,b, C.2-3), combine to give,

$$
\begin{gathered}
F_{4}=E_{1}\left[\sin \left(d_{1} l_{1}\right)-\left(d_{1} / d_{2}\right)\right. \\
\left.\sinh \left(d_{2} l_{1}\right)\right]+E_{2}\left[\cos \left(d_{1} l_{1}\right)-\cosh \left(d_{2} l_{1}\right)\right]-F_{2}= \\
=E_{1} p_{1}+E_{2} p_{2}-F_{2} .
\end{gathered}
$$

Then equation (34b), and eq. (36b,38a), together with eq.(C.1), develop to show that,

$$
K_{1}=\frac{f_{1} F_{1}+f_{2} F_{3}}{\sin \left(\eta_{\varphi} l_{1}\right)} .
$$

Applying equation (35b), and eq. (38b), gives,

$$
M_{1}=M_{2} \tan \left(\eta_{\Phi} l_{2}\right)
$$

Using equation (34d), with eq. (36b), leads to,

$$
F_{1}=\frac{1}{\sin \left(f_{1} l_{2}\right)}\left[-F_{2} \cos \left(f_{1} l_{2}\right)+F_{3}\left(\frac{f_{2}}{f_{1}}\right)^{2} \sinh \left(f_{2} l_{2}\right)+F_{4}\left(\frac{f_{2}}{f_{1}}\right)^{2} \cosh \left(f_{2} l_{2}\right)\right] \text {. }
$$

Therefore substituting C.4, into equation (C.7), provides,

$$
\begin{gathered}
F_{1}=-F_{2}\left[\frac{\cos \left(f_{1} l_{2}\right)+\left(\frac{f_{2}}{f_{1}}\right)^{2} \cosh \left(f_{2} l_{2}\right)}{\sin \left(f_{1} l_{2}\right)}\right]+F_{3}\left[\frac{\left(\frac{f_{2}}{f_{1}}\right)^{2} \sinh \left(f_{2} l_{2}\right)}{\sin \left(f_{1} l_{2}\right)}\right]+ \\
+E_{1}\left(f_{2} / f_{1}\right)^{2} \frac{\left[\cosh \left(f_{2} l_{2}\right) \sin \left(d_{1} l_{1}\right)-\left(d_{1} / d_{2}\right) \sinh \left(d_{2} l_{1}\right) \cosh \left(f_{2} l_{2}\right)\right]}{\sin \left(f_{1} l_{2}\right)}+ \\
+E_{2}\left(f_{2} / f_{1}\right)^{2} \frac{\left[\cosh \left(f_{2} l_{2}\right) \cos \left(d_{1} l_{1}\right)-\cosh \left(d_{2} l_{1}\right) \cosh \left(f_{2} l_{2}\right)\right]}{\sin \left(f_{1} l_{2}\right)}= \\
=-F_{2} p_{3}+F_{3} p_{4}+E_{1} p_{5}+E_{2} p_{6} .
\end{gathered}
$$


Then using equation (34c), together with eq. (36a), we obtain the following,

$$
\begin{gathered}
F_{1}\left(-D_{\zeta_{2}} f_{1}^{3}+\omega_{\text {out }}^{2} I_{\zeta_{2}} f_{1}\right) \cos \left(f_{1} l_{2}\right)+F_{2}\left(D_{\zeta_{2}} f_{1}^{3}-\omega_{\text {out }}^{2} I_{\zeta_{2}} f_{1}\right) \sin \left(f_{1} l_{2}\right)+ \\
+F_{3}\left(D_{\zeta_{2}} f_{2}^{3}+\omega_{\text {out }}^{2} I_{\zeta_{2}} f_{2}\right) \cosh \left(f_{2} l_{2}\right)+F_{4}\left(D_{\zeta_{2}} f_{2}^{3}+\omega_{\text {out }}^{2} I_{\zeta_{2}} f_{2}\right) \sinh \left(f_{2} l_{2}\right)= \\
=F_{1} p_{7}+F_{2} p_{8}+F_{3} p_{9}+F_{4} p_{10}=0 .
\end{gathered}
$$

Equations (C.9) and (C.4,8) provide,

$$
\begin{gathered}
F_{3}=-F_{2}\left(\frac{p_{8}-p_{3} p_{7}-p_{10}}{p_{4} p_{7}+p_{9}}\right)-E_{1}\left(\frac{p_{1} p_{10}+p_{5} p_{7}}{p_{4} p_{7}+p_{9}}\right)-E_{2}\left(\frac{p_{2} p_{10}+p_{6} p_{7}}{p_{4} p_{7}+p_{9}}\right)= \\
=F_{2} q_{8}+E_{1} p_{11}+E_{2} p_{12} .
\end{gathered}
$$

Also, using (C.8,10) in eq. ( C.5) we get,

$$
\begin{gathered}
K_{1}=\frac{F_{2}\left[f_{1}\left(q_{8} p_{4}-p_{3}\right)+f_{2} q_{8}\right]+E_{1}\left[f_{1}\left(p_{11} p_{4}+p_{5}\right)+f_{2} p_{11}\right]+E_{2}\left[f_{1}\left(p_{12} p_{4}+p_{6}\right)+f_{2} p_{12}\right]}{\sin \left(\eta_{\varphi} l_{1}\right)}= \\
=F_{2} q_{2}+E_{1} q_{4}+E_{2} q_{5} .
\end{gathered}
$$

Equation 35a and eq.(36a, 38b, C.2, C.3), gives,

$$
\begin{aligned}
M_{2}=E_{1}\left[d_{1} \cosh \left(d_{2} l_{1}\right)\right. & \left.-d_{1} \cos \left(d_{1} l_{1}\right)\right]+E_{2}\left[d_{1} \sin \left(d_{1} l_{1}\right)+d_{2} \sinh \left(d_{2} l_{1}\right)\right]=, \\
& =E_{1} p_{13}+E_{2} p_{14} .
\end{aligned}
$$

Also, taking into account equation (C.12) and so eq. (C.6) takes the final form,

$$
M_{1}=E_{1} p_{13} \tan \left(\eta_{\Phi} l_{2}\right)+E_{2} p_{14} \tan \left(\eta_{\Phi} l_{2}\right) .
$$

Equation (35a) and eq. (38b, 36a) , and also considering eq. ( C.2-3), provides the following,

$$
\begin{gathered}
M_{1}=E_{1} \frac{D_{\eta_{1}}}{D_{\xi_{2}} \eta_{\Phi}}\left[d_{1}^{2} \sin \left(d_{1} l_{1}\right)+d_{1} d_{2} \sinh \left(d_{2} l_{1}\right)\right]+E_{2} \frac{D_{\eta_{1}}}{D_{\xi_{2}} \eta_{\Phi}}\left[d_{1}^{2} \cos \left(d_{1} l_{1}\right)+d_{2}^{2} E_{2} \cosh \left(d_{2} l_{1}\right)\right]=, \\
=E_{1} p_{15}+E_{2} p_{16} .
\end{gathered}
$$

Therefore from equations $(\mathrm{C} .13,14)$ it arises that,

$$
E_{1}=E_{2}\left(\frac{p_{16}-p_{14} \tan \left(\eta_{\Phi} l_{2}\right)}{p_{13} \tan \left(\eta_{\Phi} l_{2}\right)-p_{15}}\right)=E_{2} q_{3} .
$$

So equation (C.15), with eq. ( C.14), gives,

$$
M_{1}=E_{2}\left(q_{3} p_{15}+p_{16}\right) .
$$

By considering equation (C.15) eq. (C.4) we obtain the form,

$$
F_{4}=E_{2}\left(q_{3} p_{1}+p_{2}\right)-F_{2}=q_{1} E_{2}-F_{2} .
$$

From equation (C.15) and eq. (C.10) results,

$$
F_{3}=F_{2} q_{8}+E_{2}\left(q_{3} p_{11}+p_{12}\right)=F_{2} q_{8}+E_{2} q_{9}
$$

Also, using equations (C.15 and C.18) eq. (C.8) becomes,

$$
F_{1}=F_{2}\left(q_{8} p_{4}-p_{3}\right)+E_{2}\left(q_{9} p_{4}+q_{3} p_{5}+p_{6}\right)=F_{2} q_{6}+E_{2} q_{7}
$$


It is shown that by utilising (C.15) the equation (C.11) becomes,

$$
K_{1}=F_{2} q_{2}+E_{2}\left(q_{3} q_{4}+q_{5}\right) .
$$

Equation (32c) together with eq. (36a, C.2-3,15, 38a, C.1, C.20, 36b,C.17-19), generates the following,

$$
\begin{gathered}
-I_{\zeta 2} \omega_{\text {out }}^{2}\left(F_{2} q_{2}+E_{2}\left(q_{3} q_{4}+q_{5}\right)\right) \sin \left(\eta_{\varphi} l_{1}\right)- \\
-D_{\zeta 2}\left[F_{2}\left(-q_{6} f_{1}^{3}+f_{2}^{3} q_{8}\right)+E_{2}\left(-q_{7} f_{1}^{3}+q_{9} f_{2}^{3}\right)\right]+ \\
+D_{\eta_{1}} E_{2}\left[-q_{3} d_{1}^{3} \cos \left(d_{1} l_{1}\right)+d_{1}^{3} \sin \left(d_{1} l_{1}\right)-q_{3} d_{1} d_{2}^{2} \cosh \left(d_{2} l_{1}\right)-d_{2}^{3} \sinh \left(d_{2} l_{1}\right)\right]+ \\
+\omega_{\text {out }}^{2} I_{\eta_{1}} E_{2}\left[q_{3} d_{1} \cos \left(d_{1} l_{1}\right)-q_{3} d_{1} \cosh \left(d_{2} l_{1}\right)-d_{1} \sin \left(d_{1} l_{1}\right)-d_{2} \sinh \left(d_{2} l_{1}\right)\right]=0,(\mathrm{C} .21)
\end{gathered}
$$

which, with some simple algebraic manipulation, leads to,

$$
\begin{aligned}
& F_{2}=E_{2}\left\{\frac{I_{\zeta 2} \omega_{\text {out }}^{2}\left(q_{3} q_{4}+q_{5}\right) \sin \left(\eta_{\varphi} l_{1}\right)+D_{\zeta 2}\left(-q_{7} f_{1}^{3}+f_{2}^{3} q_{9}\right)-}{-I_{\zeta 2} \omega_{\text {out }}^{2} q_{2} \sin \left(\eta_{\varphi} l_{1}\right)-D_{\zeta 2}\left(-q_{6} f_{1}^{3}+f_{2}^{3} q_{8}\right)}\right. \\
& \underline{-D_{\eta 1}\left\{-q_{3}\left[d_{1}^{3} \cos \left(d_{1} l_{1}\right)+d_{1} d_{2}^{2} \cosh \left(d_{2} l_{1}\right)\right]+d_{1}^{3} \sin \left(d_{1} l_{1}\right)-d_{2}^{3} \sinh \left(d_{2} l_{1}\right)\right\}-} \\
& \left.\underline{-I_{\eta 1} \omega_{\text {out }}^{2}\left\{q_{3}\left[d_{1} \cos \left(d_{1} l_{1}\right)-d_{1} \cosh \left(d_{2} l_{1}\right)\right]-d_{1} \sin \left(d_{1} l_{1}\right)-d_{2} \sinh \left(d_{2} l_{1}\right)\right\}}\right\}=E_{2} q_{10} .
\end{aligned}
$$

The parameters $\mathrm{p}_{\mathrm{i}}(\mathrm{i}=1, \ldots 16)$ are used in order to simplify the expressions and make the algebraic manipulations simpler and they are given by,

$$
\begin{gathered}
p_{1}=\left[\sin \left(d_{1} l_{1}\right)-\left(d_{1} d_{2}\right) \sinh \left(d_{2} l_{1}\right)\right], p_{2}=\left[\cos \left(d_{1} l_{1}\right)-\cosh \left(d_{2} l_{1}\right)\right], \\
p_{3}=\left[\frac{\cos \left(f_{1} l_{2}\right)+\left(\frac{f_{2}}{f_{1}}\right)^{2} \cosh \left(f_{2} l_{2}\right)}{\sin \left(f_{1} l_{2}\right)}\right], p_{4}=\left[\frac{\left(\frac{f_{2}}{f_{1}}\right)^{2} \sinh \left(f_{2} l_{2}\right)}{\sin \left(f_{1} l_{2}\right)}\right], \\
p_{5}=\left(f_{2} / f_{1}\right)^{2} \frac{\left[\cosh \left(f_{2} l_{2}\right) \sin \left(d_{1} l_{1}\right)-\left(d_{1} / d_{2}\right) \sinh \left(d_{2} l_{1}\right) \cosh \left(f_{2} l_{2}\right)\right]}{\sin \left(f_{1} l_{2}\right)}, \\
p_{6}=\left(f_{2} / f_{1}\right)^{2} \frac{\left[\cosh \left(f_{2} l_{2}\right) \cos \left(d_{1} l_{1}\right)-\cosh \left(d_{2} l_{1}\right) \cosh \left(f_{2} l_{2}\right)\right]}{\sin \left(f_{1} l_{2}\right)}, \\
p_{7}=\left(-D_{\zeta_{2}} f_{1}^{3}+\omega_{\text {out }}^{2} I_{\zeta_{2}} f_{1}\right) \cos \left(f_{1} l_{2}\right), p_{8}=\left(D_{\zeta_{2}} f_{1}^{3}-\omega_{\text {out }}^{2} I_{\zeta_{2}} f_{1}\right) \sin \left(f_{1} l_{2}\right), \\
\left.p_{9}=\left(D_{\zeta_{2}} f_{2}^{3}+\omega_{\text {out }}^{2} I_{\zeta_{2}} f_{2}\right) \cosh \left(f_{2} l_{2}\right), p_{10}=\left(D_{\zeta_{2}} f_{2}^{3}+\omega_{\text {out }}^{2} I_{\zeta_{2}} f_{2}\right) \sinh \left(f_{2} l_{2}\right), \quad \text { (C.24a, }\right) \\
p_{11}=-\left(\frac{p_{1} p_{10}+p_{5} p_{7}}{p_{4} p_{7}+p_{9}}\right), p_{12}=-\left(\frac{\left.p_{2} p_{10}+p_{6} p_{7}\right)}{p_{4} p_{7}+p_{9}}\right), p_{13}=\left[d_{1} \cosh \left(d_{2} l_{1}\right)-d_{1} \cos \left(d_{1} l_{1}\right)\right], \text { (C.28a,b,c) } \\
p_{14}=\left[d_{1} \sin \left(d_{1} l_{1}\right)+d_{2} \sinh \left(d_{2} l_{1}\right)\right], p_{15}=\frac{D_{\eta_{1}}}{D_{\xi_{2}} \eta_{\Phi}}\left[d_{1}^{2} \sin \left(d_{1} l_{1}\right)+d_{1} d_{2} \sinh \left(d_{2} l_{1}\right)\right], \\
p_{16}=\frac{D_{\eta_{1}}}{D_{\xi_{2}} \eta_{\Phi}}\left[d_{1}^{2} \cos \left(d_{1} l_{1}\right)+d_{2}^{2} \cosh \left(d_{2} l_{1}\right)\right] .
\end{gathered}
$$

The final definition of the constants for the mode shape equations (36) and (38) for the simplified expressions are defined through a set of parameters $q_{i}$ (with $i=1, . .10$ ) which are given by,

$$
\begin{gathered}
q_{1}=\left(q_{3} p_{1}+p_{2}\right)= \\
=\left\{\frac{D_{\eta_{1}}\left[d_{1}^{2} \cos \left(d_{1} l_{1}\right)+d_{2}^{2} \cosh \left(d_{2} l_{1}\right)\right]-D_{\xi_{2}} \eta_{\Phi} \tan \left(\eta_{\Phi} l_{2}\right)\left[d_{1} \sin \left(d_{1} l_{1}\right)+d_{2} \sinh \left(d_{2} l_{1}\right)\right]}{D_{\xi_{2}} \eta_{\Phi} \tan \left(\eta_{\Phi} l_{2}\right)\left[-d_{1} \cos \left(d_{1} l_{1}\right)+d_{1} \cosh \left(d_{2} l_{1}\right)\right]-D_{\eta 1}\left[d_{1}^{2} \sin \left(d_{1} l_{1}\right)+d_{1} d_{2} \sinh \left(d_{2} l_{1}\right)\right]}\right\} \times \\
\times\left[\sin \left(d_{1} l_{1}\right)-\left(d_{1} / d_{2}\right) \sinh \left(d_{2} l_{1}\right)\right]+\cos \left(d_{1} l_{1}\right)-\cosh \left(d_{2} l_{1}\right), \\
q_{2}=\frac{\left[q_{8}\left(f_{1} p_{4}+f_{2}\right)-p_{3} f_{1}\right]}{\sin \left(\eta_{\varphi} l_{1}\right)}
\end{gathered}
$$


$=\left\{\left[\frac{\left(D_{\zeta 2} f_{2}^{3} \sinh \left(f_{2} l_{2}\right)+I_{\zeta 2} \omega_{\text {out }}^{2} f_{2} \sinh \left(f_{2} l_{2}\right)\right) \sin \left(f_{1} l_{2}\right)-}{\sin \left(\eta_{\varphi} l_{1}\right)\left\{\left(f_{2} / f_{1}\right)^{2}\left[-D_{\zeta 2} f_{1}^{3} \cos \left(f_{1} l_{2}\right)+I_{\zeta 2} \omega_{\text {out }}^{2} f_{1} \cos \left(f_{1} l_{2}\right)\right] \sinh \left(f_{2} l_{2}\right)+\left[D_{\zeta 2} f_{2}^{3} \cosh \left(f_{2} l_{2}\right)+I_{\zeta 2} \omega_{\text {out }}^{2} f_{2} \cosh \left(f_{2} l_{2}\right)\right] \sin \left(f_{1} l_{2}\right)\right\}}\right.\right.$ $\left.\underline{-\left[D_{\zeta 2} f_{1}^{3} \sin \left(f_{1} l_{2}\right)-I_{\zeta 2} \omega_{\text {out }}^{2} f_{1} \sin \left(f_{1} l_{2}\right)\right] \sin \left(f_{1} l_{2}\right)+\left[-D_{\zeta 2} f_{1}^{3} \cos \left(f_{1} l_{2}\right)+I_{\zeta 2} \omega_{\text {out }}^{2} f_{1} \cos \left(f_{1} l_{2}\right)\right]\left[\cos \left(f_{1} l_{2}\right)+\left(f_{2} / f_{1}\right)^{2} \cosh \left(f_{2} l_{2}\right)\right]}\right] \times$

$$
\begin{gathered}
\left.\times\left[\frac{f_{1}\left(f_{2} / f_{1}\right)^{2} \sinh \left(f_{2} l_{2}\right)}{\sin \left(f_{1} l_{2}\right)}+f_{2}\right]-\frac{f_{1} \cos \left(f_{1} l_{2}\right)+f_{1}\left(f_{2} / f_{1}\right)^{2} \cosh \left(f_{2} l_{2}\right)}{\sin \left(\eta_{\varphi} l_{1}\right) \sin \left(f_{1} l_{2}\right)}\right\} \\
q_{3}=\left(\frac{p_{16}-p_{14} \tan \left(\eta_{\Phi} l_{2}\right)}{p_{13} \tan \left(\eta_{\Phi} l_{2}\right)-p_{15}}\right)= \\
=\frac{D_{\eta 1}\left[d_{1}^{2} \cos \left(d_{1} l_{1}\right)+d_{2}^{2} \cosh \left(d_{2} l_{1}\right)\right]-D_{\xi 2} \eta_{\Phi}\left[d_{1} \sin \left(d_{1} l_{1}\right)+d_{2} \sinh \left(d_{2} l_{1}\right)\right] \tan \left(\eta_{\Phi} l_{2}\right)}{D \xi_{2} \eta_{\Phi}\left[-d_{1} \cos \left(d_{1} l_{1}\right)+d_{1} \cosh \left(d_{2} l_{1}\right)\right] \tan \left(\eta_{\Phi} l_{2}\right)-D_{\eta 1}\left[d_{1}^{2} \sin \left(d_{1} l_{1}\right)+d_{1} d_{2} \sinh \left(d_{2} l_{1}\right)\right]}
\end{gathered}
$$

$$
q_{4}=\frac{\left[p_{11}\left(f_{1} p_{4}+f_{2}\right)+f_{1} p_{5}\right]}{\sin \left(\eta_{\varphi} l_{1}\right)}=
$$

$=\left\{-\left[\frac{\left[\sin \left(d_{1} l_{1}\right)-\left(\frac{d_{1}}{d_{2}}\right) \sinh \left(d_{2} l_{1}\right)\right]\left[D_{\zeta 2} f_{2}^{3} \sinh \left(f_{2} l_{2}\right)+I_{\zeta 2} \omega_{\text {out }}^{2} f_{2} \sinh \left(f_{2} l_{2}\right)\right] \sin \left(f_{1} l_{2}\right)+}{\sin \left(\eta_{\varphi} l_{1}\right)\left\{\left(f_{2} / f_{1}\right)^{2} \sinh \left(f_{2} l_{2}\right)\left[-D_{\zeta 2} f_{1}^{3} \cos \left(f_{1} l_{2}\right)+I_{\zeta 2} \omega_{\text {out }}^{2} f_{1} \cos \left(f_{1} l_{2}\right)\right]+\sin \left(f_{1} l_{2}\right)\left[D_{\zeta 2} f_{2}^{3} \cosh \left(f_{2} l_{2}\right)+I_{\zeta 2} \omega_{\text {out }}^{2} f_{2} \cosh \left(f_{2} l_{2}\right)\right]\right\}}\right.\right.$ $\left.\underline{+\left(f_{2} / f_{1}\right)^{2}\left[-D_{\zeta 2} f_{1}^{3} \cos \left(f_{1} l_{2}\right)+I_{\zeta 2} \omega_{\text {out }}^{2} f_{1} \cos \left(f_{1} l_{2}\right)\right]\left[\cosh \left(f_{2} l_{2}\right) \sin \left(d_{1} l_{1}\right)-\left(d_{1} / d_{2}\right) \sinh \left(d_{2} l_{1}\right) \cosh \left(f_{2} l_{2}\right)\right]}\right] \times$

$$
\left.\times\left[f_{1} \frac{\left(f_{2} / f_{1}\right)^{2} \sinh \left(f_{2} l_{2}\right)}{\sin \left(f_{1} l_{2}\right)}+f_{2}\right]+\frac{f_{1}\left(f_{2} / f_{1}\right)^{2}\left[\cosh \left(f_{2} l_{2}\right) \sin \left(d_{1} l_{1}\right)-\left(d_{1} / d_{2}\right) \sinh \left(d_{2} l_{1}\right) \cosh \left(f_{2} l_{2}\right)\right]}{\sin \left(\eta_{\varphi} l_{1}\right) \sin \left(f_{1} l_{2}\right)}\right\},
$$

$$
q_{5}=\frac{\left[p_{12}\left(p_{4} f_{1}+f_{2}\right)+p_{6} f_{1}\right]}{\sin \left(\eta_{\varphi} l_{1}\right)}=
$$

$=\left\{-\left[\frac{\sin \left(f_{1} l_{2}\right)\left[\cos \left(d_{1} l_{1}\right)-\cosh \left(d_{2} l_{1}\right)\right]\left[D_{\zeta 2} f_{2}^{3} \sinh \left(f_{2} l_{2}\right)+I_{\zeta 2} \omega_{\text {out }}^{2} f_{2} \sinh \left(f_{2} l_{2}\right)\right]+}{\sin \left(\eta_{\varphi} l_{1}\right)\left\{\left(f_{2} / f_{1}\right)^{2} \sinh \left(f_{2} l_{2}\right)\left[-D_{\zeta 2} f_{1}^{3} \cos \left(f_{1} l_{2}\right)+I_{\zeta 2} \omega_{\text {out }}^{2} f_{1} \cos \left(f_{1} l_{2}\right)\right]+\sin \left(f_{1} l_{2}\right)\left[D_{\zeta 2} f_{2}^{3} \cosh \left(f_{2} l_{2}\right)+I_{\zeta 2} \omega_{\text {out }}^{2} f_{2} \cosh \left(f_{2} l_{2}\right)\right]\right\}}\right.\right.$

$\left.\underline{+\left(f_{2} / f_{1}\right)^{2}\left[-D_{\zeta 2} f_{1}^{3} \cos \left(f_{1} l_{2}\right)+I_{\zeta 2} \omega_{\text {out }}^{2} f_{1} \cos \left(f_{1} l_{2}\right)\right]\left[\cosh \left(f_{2} l_{2}\right) \cos \left(d_{1} l_{1}\right)-\cosh \left(d_{2} l_{1}\right) \cosh \left(f_{2} l_{2}\right)\right]}\right] \times \times$

$$
\left.\left[\frac{f_{1}\left(f_{2} / f_{1}\right)^{2} \sinh \left(f_{2} l_{2}\right)}{\sin \left(f_{1} l_{2}\right)}+f_{2}\right]+\frac{f_{1}\left(f_{2} / f_{1}\right)^{2}\left[\cosh \left(f_{2} l_{2}\right) \cos \left(d_{1} l_{1}\right)-\cosh \left(d_{2} l_{1}\right) \cosh \left(f_{2} l_{2}\right)\right]}{\sin \left(\eta_{\varphi} l_{1}\right) \sin \left(f_{1} l_{2}\right)}\right\}
$$

$$
q_{6}=\left(q_{8} p_{4}-p_{3}\right)=-\left[\frac{\left(\frac{f_{2}}{f_{1}}\right)^{2} \sinh \left(f_{2} l_{2}\right)}{\sin \left(f_{1} l_{2}\right)}\right] \times
$$

$\times\left\{\frac{\left[D_{\zeta 2} f_{1}^{3} \sin \left(f_{1} l_{2}\right)-I_{\zeta 2} \omega_{\text {out }}^{2} f_{1} \sin \left(f_{1} l_{2}\right)\right] \sin \left(f_{1} l_{2}\right)-\left[-D_{\zeta 2} f_{1}^{3} \cos \left(f_{1} l_{2}\right)+I_{\zeta 2} \omega_{\text {out }}^{2} f_{1} \cos \left(f_{1} l_{2}\right)\right]\left[\cos \left(f_{1} l_{2}\right)+\left(\frac{f_{2}}{f_{1}}\right)^{2} \cosh \left(f_{2} l_{2}\right)\right]}{\left(f_{2} / f_{1}\right)^{2}\left[-D_{\zeta 2} f_{1}^{3} \cos \left(f_{1} l_{2}\right)+I_{\zeta 2} \omega_{\text {out }}^{2} f_{1} \cos \left(f_{1} l_{2}\right)\right] \sinh \left(f_{2} l_{2}\right)+\left[D_{\zeta 2} f_{2}^{3} \cosh \left(f_{2} l_{2}\right)+I_{\zeta 2} \omega_{\text {out }}^{2} f_{2} \cosh \left(f_{2} l_{2}\right)\right] \sin \left(f_{1} l_{2}\right)}\right.$

$$
\begin{gathered}
\left.\frac{-\left[D_{\zeta 2} f_{2}^{3} \sinh \left(f_{2} l_{2}\right)+I_{\zeta 2} \omega_{\text {out }}^{2} f_{2} \sinh \left(f_{2} l_{2}\right)\right] \sin \left(f_{1} l_{2}\right)}{-}\right\}-\frac{\cos \left(f_{1} l_{2}\right)+\left(f_{2} / f_{1}\right)^{2} \cosh \left(f_{2} l_{2}\right)}{\sin \left(f_{1} l_{2}\right)}, \\
q_{7}=\left(q_{9} p_{4}+q_{3} p_{5}+p_{6}\right)=
\end{gathered}
$$

$=q_{3}\left\{\frac{\left(\frac{f_{2}}{f_{1}}\right)^{2}\left[\cosh \left(f_{2} l_{2}\right) \sin \left(d_{1} l_{1}\right)-\left(\frac{d_{1}}{d_{2}}\right) \sinh \left(d_{2} l_{1}\right) \cosh \left(f_{2} l_{2}\right)\right]}{\sin \left(f_{1} l_{2}\right)}\right\}+\left\{\frac{\left(\frac{f_{2}}{f_{1}}\right)^{2}\left[\cosh \left(f_{2} l_{2}\right) \cos \left(d_{1} l_{1}\right)-\cosh \left(d_{2} l_{1}\right) \cosh \left(f_{2} l_{2}\right)\right]}{\sin \left(f_{1} l_{2}\right)}\right\}-$ 


$$
-q_{3} \times\left\{\frac{\left(\frac{f_{2}}{f_{1}}\right)^{2} \sinh \left(f_{2} l_{2}\right)}{\sin \left(f_{1} l_{2}\right)}\right\} \times
$$

$\times\left\{\frac{\left[\sin \left(d_{1} l_{1}\right)-\left(\frac{d_{1}}{d_{2}}\right) \sinh \left(d_{2} l_{1}\right)\right]\left[D_{\zeta 2} f_{2}^{3} \sinh \left(f_{2} l_{2}\right)+I_{\zeta 2} \omega_{o u t}^{2} f_{2} \sinh \left(f_{2} l_{2}\right)\right] \sin \left(f_{1} l_{2}\right)+}{\left(f_{2} / f_{1}\right)^{2} \sinh \left(f_{2} l_{2}\right)\left[-D_{\zeta 2} f_{1}^{3} \cos \left(f_{1} l_{2}\right)+I_{\zeta 2} \omega_{o u t}^{2} f_{1} \cos \left(f_{1} l_{2}\right)\right]+\sin \left(f_{1} l_{2}\right)\left[D_{\zeta 2} f_{2}^{3} \cosh \left(f_{2} l_{2}\right)+I_{\zeta 2} \omega_{\text {out }}^{2} f_{2} \cosh \left(f_{2} l_{2}\right)\right]}\right.$ $\left.\frac{+\left(f_{2} / f_{1}\right)^{2}\left[-D_{\zeta 2} f_{1}^{3} \cos \left(f_{1} l_{2}\right)+I_{\zeta 2} \omega_{o u t}^{2} f_{1} \cos \left(f_{1} l_{2}\right)\right]\left[\cosh \left(f_{2} l_{2}\right) \sin \left(d_{1} l_{1}\right)-\left(d_{1} / d_{2}\right) \sinh \left(d_{2} l_{1}\right) \cosh \left(f_{2} l_{2}\right)\right]}{-}\right\}-$

$$
-\left\{\frac{\left(\frac{f_{2}}{f_{1}}\right)^{2} \sinh \left(f_{2} l_{2}\right)}{\sin \left(f_{1} l_{2}\right)}\right\} \times
$$

$\times\left\{\frac{\sin \left(f_{1} l_{2}\right)\left(\cos \left(d_{1} l_{1}\right)-\cosh \left(d_{2} l_{1}\right)\right)\left[D_{\zeta 2} f_{2}^{3} \sinh \left(f_{2} l_{2}\right)+I_{\zeta 2} \omega^{2} f_{2} \sinh \left(f_{2} l_{2}\right)\right]+}{\left(f_{2} / f_{1}\right)^{2} \sinh \left(f_{2} l_{2}\right)\left[-D_{\zeta 2} f_{1}^{3} \cos \left(f_{1} l_{2}\right)+I_{\zeta 2} \omega^{2} f_{1} \cos \left(f_{1} l_{2}\right)\right]+\sin \left(f_{1} l_{2}\right)\left[D_{\zeta 2} f_{2}^{3} \cosh \left(f_{2} l_{2}\right)+I_{\zeta 2} \omega^{2} f_{2} \cosh \left(f_{2} l_{2}\right)\right]}\right.$ $\left.\underline{+\left[-D_{\zeta 2} f_{1}^{3} \cos \left(f_{1} l_{2}\right)+I_{\zeta 2} \omega^{2} f_{1} \cos \left(f_{1} l_{2}\right)\right]\left(f_{2} / f_{1}\right)^{2}\left[\cosh \left(f_{2} l_{2}\right) \cos \left(d_{1} l_{1}\right)-\cosh \left(d_{2} l_{1}\right) \cosh \left(f_{2} l_{2}\right)\right]}\right\}$,

$$
\begin{gathered}
q_{8}=-\left(\frac{p_{8}-p_{3} p_{7}-p_{10}}{p_{4} p_{7}+p_{9}}\right)= \\
=-\left\{\frac{\left[D_{\zeta 2} f_{1}^{3}-I_{\zeta 2} \omega_{\text {out }}^{2} f_{1}\right] \sin \left(f_{1} l_{2}\right) \sin \left(f_{1} l_{2}\right)-\cos \left(f_{1} l_{2}\right)\left[-D_{\zeta 2} f_{1}^{3}+I_{\zeta 2} \omega_{\text {out }}^{2} f_{1}\right]\left[\cos \left(f_{1} l_{2}\right)+\left(\frac{f_{2}}{f_{1}}\right)^{2} \cosh \left(f_{2} l_{2}\right)\right]-}{\left(f_{2} / f_{1}\right)^{2} \sinh \left(f_{2} l_{2}\right) \cos \left(f_{1} l_{2}\right)\left[-D_{\zeta 2} f_{1}^{3}+I_{\zeta 2} \omega_{\text {out }}^{2} f_{1}\right]+\left[D_{\zeta 2} f_{2}^{3}+I_{\zeta 2} \omega_{\text {out }}^{2} f_{2}\right] \cosh \left(f_{2} l_{2}\right) \sin \left(f_{1} l_{2}\right)}\right. \\
\frac{-\left(D_{\zeta 2} f_{2}^{3}+I_{\zeta 2} \omega_{\text {out }}^{2} f_{2}\right) \sinh \left(f_{2} l_{2}\right) \sin \left(f_{1} l_{2}\right)}{q_{9}=q_{3} p_{11}+p_{12}=} \\
=-q_{3}\left\{\frac{\left[\operatorname{C.37}\left(d_{1} l_{1}\right)-\left(\frac{d_{1}}{d_{2}}\right) \sinh \left(d_{2} l_{1}\right)\right]\left[D_{\zeta 2} f_{2}^{3}+I_{\zeta 2} \omega_{\text {out }}^{2} f_{2}\right] \sinh \left(f_{2} l_{2}\right) \sin \left(f_{1} l_{2}\right)+}{\left(f_{2} / f_{1}\right)^{2} \sinh \left(f_{2} l_{2}\right) \cos \left(f_{1} l_{2}\right)\left[-D_{\zeta 2} f_{1}^{3}+I_{\zeta 2} \omega_{\text {out }}^{2} f_{1}\right]+\sin \left(f_{1} l_{2}\right) \cosh \left(f_{2} l_{2}\right)\left[D_{\zeta 2} f_{2}^{3}+I_{\zeta 2} \omega_{\text {out }}^{2} f_{2}\right]}\right.
\end{gathered}
$$

$\left.\underline{+\left(f_{2} / f_{1}\right)^{2} \cos \left(f_{1} l_{2}\right)\left[-D_{\zeta 2} f_{1}^{3}+I_{\zeta 2} \omega_{\text {out }}^{2} f_{1}\right]\left[\cosh \left(f_{2} l_{2}\right) \sin \left(d_{1} l_{1}\right)-\left(d_{1} / d_{2}\right) \sinh \left(d_{2} l_{1}\right) \cosh \left(f_{2} l_{2}\right)\right]}\right\}$

$$
\begin{gathered}
-\left\{\frac{\sinh \left(f_{2} l_{2}\right) \sin \left(f_{1} l_{2}\right)\left[\cos \left(d_{1} l_{1}\right)-\cosh \left(d_{2} l_{1}\right)\right]\left[D_{\zeta 2} f_{2}^{3}+I_{\zeta 2} \omega_{\text {out }}^{2} f_{2}\right]+}{\left(f_{2} / f_{1}\right)^{2} \sinh \left(f_{2} l_{2}\right)\left[\cos \left(f_{1} l_{2}\right)-D_{\zeta 2} f_{1}^{3}+I_{\zeta 2} \omega_{\text {out }}^{2} f_{1}\right]+\sin \left(f_{1} l_{2}\right) \cosh \left(f_{2} l_{2}\right)\left[D_{\zeta 2} f_{2}^{3}+I_{\zeta 2} \omega_{\text {out }}^{2} f_{2}\right]}\right. \\
\left.\frac{\left.+\left(f_{2} / f_{1}\right)^{2} \cos \left(f_{1} l_{2}\right)\left[-D_{\zeta 2} f_{1}^{3}+I_{\zeta 2} \omega_{\text {out }}^{2} f_{1}\right]\left[\cosh \left(f_{2} l_{2}\right) \cos \left(d_{1} l_{1}\right)-\cosh \left(d_{2} l_{1}\right) \cosh \left(f_{2} l_{2}\right)\right]\right]}{\text { a }}\right\},(\mathrm{C} .38) \\
q_{10}=\frac{I_{\zeta 2} \omega_{\text {out }}^{2}\left(q_{3} q_{4}+q_{5}\right) \sin \left(\eta_{\varphi} l_{1}\right)+D_{\zeta 2}\left(-q_{7} f_{1}^{3}+f_{2}^{3} q_{9}\right)-}{-I_{\zeta 2} \omega_{\text {out }}^{2} q_{2} \sin \left(\eta_{\varphi} l_{1}\right)-D_{\zeta 2}\left(-q_{6} f_{1}^{3}+f_{2}^{3} q_{8}\right)} \\
-D_{\eta 1}\left\{-q_{3}\left[d_{1}^{3} \cos \left(d_{1} l_{1}\right)+d_{1} d_{2}^{2} \cosh \left(d_{2} l_{1}\right)\right]+d_{1}^{3} \sin \left(d_{1} l_{1}\right)-d_{2}^{3} \sinh \left(d_{2} l_{1}\right)\right\}-
\end{gathered}
$$$$
\underline{-I_{\eta 1} \omega_{\text {out }}^{2}\left\{q_{3}\left[d_{1} \cos \left(d_{1} l_{1}\right)-d_{1} \cosh \left(d_{2} l_{1}\right)\right]-d_{1} \sin \left(d_{1} l_{1}\right)-d_{2} \sinh \left(d_{2} l_{1}\right)\right\}} .
$$ 


\section{TABLE CAPTIONS}

Table 1.1. In-plane bending of beam 2 model-1.

Table 1.2. Out-of-plane bending of beam 2 model-1.

Table 1.3. In-plane bending of beam 2 model-2.

Table 1.4. Out-of-plane bending of beam 2 model-2.

Table 1.5. In-plane motion, model 1.

Table 1.6. In-plane motion, model 2.

Table 1.7. In-plane motion, model 3.

Table 1.8. In-plane motion, model 4.

Table 1.9. Out-of-plane motion, model 1.

Table 1.10. Out-of-plane motion, model 2.

Table 1.11. Out-of-plane motion, model 3.

Table 1.12. Out-of-plane motion, model 4. 


\section{TABLES}

Table 1.1.

\begin{tabular}{|ccccccc|}
\hline Mode & $\begin{array}{c}\text { Freq. } \\
\text { FE } \\
\mathbf{( H z )}\end{array}$ & $\begin{array}{c}\text { Freq., } \\
\text { Theory } \\
\text { without } \\
\text { InertiaTerms } \\
\mathbf{( H z )}\end{array}$ & $\begin{array}{c}\text { Freq., } \\
\text { Theory, with } \\
\text { InertiaTerms } \\
\mathbf{( H z )}\end{array}$ & $\begin{array}{c}\text { \% Rel.Dif. } \\
\text { with } \\
\text { Inertia } \\
\text { effect } \\
\text { (theory) }\end{array}$ & $\begin{array}{c}\text { \% Rel. Dif. } \\
\text { FE with } \\
\text { theory } \\
\text { (inertia) }\end{array}$ & $\begin{array}{c}\text { MAC } \\
\text { FE with } \\
\text { theory } \\
\text { (inertia) }\end{array}$ \\
\hline 1 & 107.7 & 107.7 & 107.7 & 0.0 & 0.0 & 1.000 \\
2 & 673.4 & 674.9 & 674.5 & -0.1 & 0.2 & 1.000 \\
3 & 1879.6 & 1889.7 & 1887.3 & -0.1 & 0.4 & 1.000 \\
4 & 3667.0 & 3703.2 & 3694.4 & -0.2 & 0.7 & 1.000 \\
5 & 6027.3 & 6121.6 & 6098.4 & -0.4 & 1.2 & 1.000 \\
\hline
\end{tabular}

Table 1.2.

\begin{tabular}{|ccccccc|}
\hline Mode & $\begin{array}{c}\text { Freq. FE } \\
\text { (Hz) }\end{array}$ & $\begin{array}{c}\text { Freq., } \\
\text { Theory } \\
\text { without } \\
\text { Inertia } \\
\text { Terms } \\
\text { (Hz) }\end{array}$ & $\begin{array}{c}\text { Freq., } \\
\text { Theory, with } \\
\text { Inertia Terms } \\
\text { (Hz) }\end{array}$ & $\begin{array}{c}\text { \% Rel.Dif. } \\
\text { with } \\
\text { Inertia } \\
\text { effect } \\
\text { (theory) }\end{array}$ & $\begin{array}{c}\text { \% Rel. Dif. } \\
\text { FE with } \\
\text { theory } \\
\text { (inertia) }\end{array}$ & $\begin{array}{c}\text { MAC } \\
\text { FE with } \\
\text { theory } \\
\text { (inertia) }\end{array}$ \\
\hline 1 & 143.5 & 143.6 & 143.6 & 0.0 & 0.1 & \\
2 & 896.3 & 899.9 & 899.0 & -0.1 & 0.3 & 1.000 \\
3 & 2496.0 & 2519.7 & 2513.9 & -0.2 & 0.7 & 1.000 \\
4 & 4853.2 & 4937.5 & 4916.8 & -0.4 & 1.3 & 1.000 \\
5 & 7943.9 & 8162.1 & 8107.5 & -0.7 & 2.0 & 1.000 \\
\hline
\end{tabular}

Table 1.3.

\begin{tabular}{|ccccccc|}
\hline Mode & $\begin{array}{c}\text { Freq. FE } \\
\mathbf{( H z )}\end{array}$ & $\begin{array}{c}\text { Freq., } \\
\text { Theory } \\
\text { without } \\
\text { Inertia } \\
\text { Terms } \\
\mathbf{( H z )}\end{array}$ & $\begin{array}{c}\text { Freq., } \\
\text { Theory, with } \\
\text { Inertia Terms } \\
\mathbf{( H z )}\end{array}$ & $\begin{array}{c}\text { \% Rel.Dif. } \\
\text { with } \\
\text { Inertia } \\
\text { effect } \\
\text { (theory) }\end{array}$ & $\begin{array}{c}\text { \% Rel. Dif. } \\
\text { FE with } \\
\text { theory } \\
\text { (inertia) }\end{array}$ & $\begin{array}{c}\text { MAC } \\
\text { FE with } \\
\text { theory } \\
\text { (inertia) }\end{array}$ \\
\hline 1 & 39.6 & 39.6 & 39.6 & 0.0 & 0.0 & \\
2 & 247.8 & 247.9 & 247.9 & 0.0 & 0.0 & 1.000 \\
3 & 693.2 & 694.2 & 694.0 & 0.0 & 0.1 & 1.000 \\
4 & 1356.7 & 1360.3 & 1359.5 & -0.1 & 0.2 & 1.000 \\
5 & 2239.2 & 2248.7 & 2246.5 & -0.1 & 0.3 & 1.000 \\
\hline
\end{tabular}


Table 1.4.

\begin{tabular}{|ccccccc|}
\hline Mode & $\begin{array}{c}\text { Freq. FE } \\
\text { (Hz) }\end{array}$ & $\begin{array}{c}\text { Freq., } \\
\text { Theory } \\
\text { without } \\
\text { Inertia }\end{array}$ & $\begin{array}{c}\text { Freq., Theory, } \\
\text { with Inertia } \\
\text { Terms } \\
\mathbf{( H z )}\end{array}$ & $\begin{array}{c}\text { \% Rel.Dif. } \\
\text { with } \\
\text { Inertia } \\
\text { effect } \\
\text { (theory) }\end{array}$ & $\begin{array}{c}\text { \% Rel. Dif. } \\
\text { FE with } \\
\text { theory } \\
\text { (inertia) }\end{array}$ & $\begin{array}{c}\text { MAC } \\
\text { FE with } \\
\text { theory } \\
\text { (inertia) }\end{array}$ \\
\hline 1 & 236.5 & $\begin{array}{c}\mathbf{H z} \text { ) } \\
237.2\end{array}$ & 237.0 & -0.1 & 0.2 & 1.000 \\
2 & 1455.8 & 1486.4 & 1478.8 & -0.5 & 1.6 & 1.000 \\
3 & 3967.8 & 4161.9 & 4111.9 & -1.2 & 3.5 & 0.999 \\
4 & 7497.0 & 8155.7 & 7977.1 & -2.2 & 6.0 & 0.997 \\
5 & 11874.0 & 13482.0 & 13019.7 & -3.6 & 8.8 & 0.993 \\
\hline
\end{tabular}

Table 1.5.

\begin{tabular}{|ccccccccc|}
\hline Mode & $\begin{array}{c}\text { FE } \\
\text { Mode }\end{array}$ & $\begin{array}{c}\text { Freq., } \\
\text { FE-a, B31 } \\
\mathbf{( H z )}\end{array}$ & $\begin{array}{c}\text { Freq., } \\
\text { FE-b, S4 } \\
\mathbf{( H z )}\end{array}$ & $\begin{array}{c}\text { Freq., } \\
\text { Theory } \\
\text { (Hz) }\end{array}$ & $\begin{array}{c}\text { \% rel. diff. } \\
\text { theory with }\end{array}$ & $\begin{array}{c}\text { \% rel. diff. } \\
\text { theory with }\end{array}$ & $\begin{array}{c}\text { MAC } \\
\text { theory } \\
\text { with FE-a }\end{array}$ & $\begin{array}{c}\text { MAC } \\
\text { theory } \\
\text { with FE-b }\end{array}$ \\
1 & 1 & 25.8 & 25.8 & 25.8 & 0.0 & 0.0 & 1.00 & 1.00 \\
2 & 3 & 77.9 & 78.0 & 78.0 & 0.1 & 0.0 & 1.00 & 1.00 \\
3 & 5 & 337.2 & 337.5 & 337.6 & 0.1 & 0.0 & 1.00 & 1.00 \\
4 & 7 & 573.5 & 573.8 & 575.2 & 0.3 & 0.2 & 1.00 & 1.00 \\
5 & 9 & 998.2 & 999.3 & 1001.7 & 0.3 & 0.2 & 1.00 & 1.00 \\
6 & 11 & 1628.0 & 1629.0 & 1639.1 & 0.7 & 0.6 & 1.00 & 1.00 \\
7 & 12 & 2032.4 & 2035.2 & 2048.5 & 0.8 & 0.6 & 1.00 & 1.00 \\
8 & 15 & 3102.7 & 3106.8 & 3133.7 & 1.0 & 0.9 & 1.00 & 1.00 \\
9 & 16 & 3529.2 & 3534.1 & 3596.6 & 1.9 & 1.7 & 1.00 & 1.00 \\
10 & 20 & 4828.8 & 4839.2 & 4896.6 & 1.4 & 1.2 & 1.00 & 1.00 \\
\hline
\end{tabular}

Table 1.6.

\begin{tabular}{|c|c|c|c|c|c|c|c|c|}
\hline Mode & $\begin{array}{c}\text { FE } \\
\text { Mode }\end{array}$ & $\begin{array}{c}\text { Freq., } \\
\text { FE-c, B31 } \\
\text { (Hz) }\end{array}$ & $\begin{array}{c}\text { Freq., } \\
\text { FE-d, S4 } \\
\text { (Hz) }\end{array}$ & $\begin{array}{c}\text { Freq., } \\
\text { Theory } \\
(\mathrm{Hz})\end{array}$ & $\begin{array}{c}\text { \% rel. diff. } \\
\text { theory with } \\
\text { FE-C }\end{array}$ & $\begin{array}{c}\% \text { rel. diff. } \\
\text { theory } \\
\text { with FE-d }\end{array}$ & $\begin{array}{c}\text { MAC } \\
\text { theory } \\
\text { with FE-c }\end{array}$ & $\begin{array}{c}\text { MAC } \\
\text { theory } \\
\text { with FE-d }\end{array}$ \\
\hline 1 & 1 & 25.8 & 25.8 & 25.9 & 0.4 & 0.4 & 0.96 & 1.00 \\
\hline 2 & 4 & 84.8 & 82.3 & 84.8 & 0.0 & 2.9 & 0.99 & 1.00 \\
\hline 3 & 5 & 365.4 & 359.4 & 365.9 & 0.1 & 1.8 & 0.99 & 1.00 \\
\hline 4 & 8 & 697.7 & 692.4 & 700.4 & 0.4 & 1.1 & 1.00 & 1.00 \\
\hline 5 & 9 & 1053.9 & 1029.5 & 1058.6 & 0.4 & 2.7 & 1.00 & 1.00 \\
\hline 6 & 12 & 1894.7 & 1892.7 & 1906.8 & 0.6 & 0.7 & 1.00 & 1.00 \\
\hline 7 & 13 & 2275.7 & 2228.1 & 2307.0 & 1.4 & 3.4 & 1.00 & 0.99 \\
\hline 8 & 15 & 3299.4 & 3264.0 & 3335.0 & 1.1 & 2.1 & 0.99 & 0.99 \\
\hline 9 & 18 & 4284.6 & 4268.9 & 4412.1 & 2.9 & 3.2 & 0.99 & 0.97 \\
\hline 10 & 20 & 4997.5 & 4930.8 & 5126.0 & 2.5 & 3.8 & 0.98 & 0.95 \\
\hline
\end{tabular}


Table 1.7.

\begin{tabular}{|ccccccccc|}
\hline Mode & $\begin{array}{c}\text { FE } \\
\text { Mode }\end{array}$ & $\begin{array}{c}\text { Freq., } \\
\text { FE-e, B31 } \\
\mathbf{( H z )}\end{array}$ & $\begin{array}{c}\text { Freq., } \\
\text { FE-f, S4 } \\
\mathbf{( H z )}\end{array}$ & $\begin{array}{c}\text { Freq., } \\
\text { Theory } \\
\text { (Hz) }\end{array}$ & $\begin{array}{c}\text { \% rel. diff. \% rel. diff. } \\
\text { theory with } \\
\text { FE-e }\end{array}$ & $\begin{array}{c}\text { MAC } \\
\text { theory } \\
\text { with FE-f } \\
\text { theory } \\
\text { with FE-e }\end{array}$ & $\begin{array}{c}\text { MAC } \\
\text { theory } \\
\text { with }\end{array}$ \\
1 & & 15.2 & 15.3 & 15.2 & 0.0 & -0.7 & 1.00 & 1.00 \\
2 & 1 & 15.2 & 41.6 & 41.4 & 0.0 & -0.5 & 1.00 & 1.00 \\
3 & 3 & 41.4 & 41.6 & 192.4 & 0.1 & -0.1 & 1.00 & 1.00 \\
4 & 4 & 192.2 & 192.6 & 192.4 & -0.6 & 1.00 & 1.00 \\
5 & 6 & 321.1 & 323.2 & 321.4 & 0.1 & -0.6 & 1.00 & 1.00 \\
6 & 7 & 612.2 & 613.8 & 613.2 & 0.2 & -0.1 & 1.00 \\
7 & 8 & 878.9 & 884.6 & 881.0 & 0.2 & -0.4 & 1.00 & 1.00 \\
8 & 11 & 1258.6 & 1263.8 & 1263.3 & 0.4 & 0.0 & 1.00 & 1.00 \\
9 & 12 & 1722.6 & 1734.7 & 1729.5 & 0.4 & -0.3 & 1.00 & 1.00 \\
10 & 14 & 2132.2 & 2145.0 & 2146.0 & 0.6 & 0.0 & 1.00 & 1.00 \\
& 16 & 2842.5 & 2864.8 & 2860.2 & 0.6 & -0.2 & 1.00 & 1.00 \\
\hline
\end{tabular}

Table 1.8.

\begin{tabular}{|c|c|c|c|c|c|c|c|c|}
\hline Mode & $\begin{array}{c}\text { FE } \\
\text { Mode }\end{array}$ & $\begin{array}{c}\text { Freq., } \\
\text { FE-h, B31 } \\
\text { (Hz) }\end{array}$ & $\begin{array}{c}\text { Freq., } \\
\text { FE-g, S4 } \\
\text { (Hz) }\end{array}$ & $\begin{array}{l}\text { Freq., } \\
\text { Theory } \\
(\mathrm{Hz})\end{array}$ & $\begin{array}{l}\text { \% rel. diff. } \\
\text { theory with } \\
\text { FE-g }\end{array}$ & $\begin{array}{c}\text { \% rel. diff. } \\
\text { theory } \\
\text { with FE-h }\end{array}$ & $\begin{array}{c}\text { MAC } \\
\text { theory } \\
\text { with FE-g }\end{array}$ & $\begin{array}{c}\text { MAC } \\
\text { theory } \\
\text { with FE-h }\end{array}$ \\
\hline 1 & 1 & 15.9 & 16.0 & 15.9 & 0.0 & -0.6 & 0.98 & 1.00 \\
\hline 2 & 3 & 55.4 & 57.0 & 55.4 & 0.0 & -2.9 & 0.98 & 1.00 \\
\hline 3 & 6 & 360.1 & 373.2 & 360.5 & 0.1 & -3.5 & 0.99 & 1.00 \\
\hline 4 & 8 & 927.4 & 946.2 & 931.3 & 0.4 & -1.6 & 0.98 & 0.98 \\
\hline 5 & 9 & 1070.6 & 1090.4 & 1085.0 & 1.3 & -0.5 & 1.00 & 0.97 \\
\hline 6 & 13 & 1857.1 & 1930.3 & 1867.2 & 0.5 & -3.4 & 0.96 & 0.99 \\
\hline 7 & 16 & 3005.0 & 3074.7 & 3037.5 & 1.1 & -1.2 & 0.94 & 0.90 \\
\hline 8 & 18 & 3278.5 & 3339.7 & 3430.6 & 4.4 & 2.6 & 0.99 & 0.88 \\
\hline 9 & 21 & 4526.9 & 4718.6 & 4586.9 & 1.3 & -2.9 & 0.91 & 0.99 \\
\hline 10 & 25 & 5965.4 & 5968.4 & 6356.6 & 6.2 & 6.1 & 0.12 & 0.11 \\
\hline
\end{tabular}

Table 1.9.

\begin{tabular}{|c|c|c|c|c|c|c|c|c|}
\hline Mode & $\begin{array}{c}\text { FE } \\
\text { Mode }\end{array}$ & $\begin{array}{c}\text { Freq., } \\
\text { FE-a, B31 } \\
\text { (Hz) }\end{array}$ & $\begin{array}{c}\text { Freq., } \\
\text { FE-b, S4 } \\
\text { (Hz) }\end{array}$ & $\begin{array}{l}\text { Freq., } \\
\text { Theory } \\
(\mathrm{Hz})\end{array}$ & $\begin{array}{l}\text { \% rel. diff. } \\
\text { theory with } \\
\text { FE-a }\end{array}$ & $\begin{array}{c}\% \text { rel. diff. } \\
\text { theory } \\
\text { with FE-b }\end{array}$ & $\begin{array}{c}\text { MAC } \\
\text { theory } \\
\text { with FE-a }\end{array}$ & $\begin{array}{c}\text { MAC } \\
\text { theory } \\
\text { with FE-b }\end{array}$ \\
\hline 1 & 2 & 30.2 & 30.3 & 30.2 & 0.0 & -0.3 & 1.00 & 1.00 \\
\hline 2 & 4 & 79.4 & 79.5 & 79.4 & 0.0 & -0.1 & 1.00 & 1.00 \\
\hline 3 & 6 & 407.1 & 408.3 & 407.7 & 0.1 & -0.1 & 1.00 & 1.00 \\
\hline 4 & 8 & 787.3 & 788.8 & 788.4 & 0.1 & -0.1 & 1.00 & 1.00 \\
\hline 5 & 10 & 1285.5 & 1289.8 & 1290.2 & 0.4 & 0.0 & 1.00 & 1.00 \\
\hline 6 & 13 & 2176.8 & 2182.2 & 2187.4 & 0.5 & 0.2 & 1.00 & 1.00 \\
\hline 7 & 14 & 2643.2 & 2652.1 & 2660.0 & 0.6 & 0.3 & 1.00 & 1.00 \\
\hline 8 & 17 & 3981.1 & 3990.6 & 4011.0 & 0.7 & 0.5 & 1.00 & 1.00 \\
\hline 9 & 18 & 4384.5 & 4385.5 & 4406.1 & 0.5 & 0.5 & 1.00 & 1.00 \\
\hline 10 & 19 & 4791.2 & 4802.0 & 4833.0 & 0.9 & 0.6 & 1.00 & 1.00 \\
\hline
\end{tabular}


Table 1.10.

\begin{tabular}{|c|c|c|c|c|c|c|c|c|}
\hline Mode & $\begin{array}{c}\text { FE } \\
\text { Mode }\end{array}$ & $\begin{array}{c}\text { Freq., } \\
\text { FE-c, B31 } \\
\text { (Hz) }\end{array}$ & $\begin{array}{c}\text { Freq., } \\
\text { FE-d, S4 } \\
\text { (Hz) }\end{array}$ & $\begin{array}{c}\text { Freq., } \\
\text { Theory } \\
(\mathrm{Hz})\end{array}$ & $\begin{array}{c}\text { \% rel. diff. } \\
\text { theory with } \\
\text { FE-c }\end{array}$ & $\begin{array}{c}\text { \% rel. diff. } \\
\text { theory } \\
\text { with FE-d }\end{array}$ & $\begin{array}{c}\text { MAC } \\
\text { theory } \\
\text { with FE-c }\end{array}$ & $\begin{array}{c}\text { MAC } \\
\text { theory } \\
\text { with FE-d }\end{array}$ \\
\hline 1 & 2 & 29.8 & 29.9 & 29.8 & 0.0 & -0.3 & 1.00 & 1.00 \\
\hline 2 & 3 & 77.7 & 77.9 & 77.7 & 0.0 & -0.3 & 1.00 & 1.00 \\
\hline 3 & 6 & 393.3 & 394.4 & 393.9 & 0.2 & -0.1 & 1.00 & 1.00 \\
\hline 4 & 7 & 629.7 & 630.4 & 630.4 & 0.1 & 0.0 & 1.00 & 1.00 \\
\hline 5 & 10 & 1246.8 & 1250.3 & 1251.1 & 0.3 & 0.1 & 1.00 & 1.00 \\
\hline 6 & 11 & 1733.3 & 1735.1 & 1738.4 & 0.3 & 0.2 & 1.00 & 1.00 \\
\hline 7 & 14 & 2538.6 & 2545.4 & 2555.2 & 0.6 & 0.4 & 1.00 & 1.00 \\
\hline 8 & 16 & 3390.3 & 3394.6 & 3409.5 & 0.6 & 0.4 & 1.00 & 1.00 \\
\hline 9 & 17 & 4056.8 & 3993.3 & 4079.2 & 0.5 & 2.1 & 1.00 & 1.00 \\
\hline 10 & 19 & 4638.3 & 4583.8 & 4667.8 & 0.6 & 1.8 & 1.00 & 1.00 \\
\hline
\end{tabular}

Table 1.11.

\begin{tabular}{|c|c|c|c|c|c|c|c|c|}
\hline Mode & $\begin{array}{c}\text { FE } \\
\text { Mode }\end{array}$ & $\begin{array}{c}\text { Freq., } \\
\text { FE-e, B31 } \\
\text { (Hz) }\end{array}$ & $\begin{array}{c}\text { Freq., } \\
\text { FE-f, S4 } \\
\text { (Hz) }\end{array}$ & $\begin{array}{c}\text { Freq., } \\
\text { Theory } \\
\text { (Hz) }\end{array}$ & $\begin{array}{l}\% \text { rel. diff. } \\
\text { theory } \\
\text { with FE-e }\end{array}$ & $\begin{array}{l}\% \text { rel. diff. } \\
\text { theory } \\
\text { with FE-f }\end{array}$ & $\begin{array}{c}\text { MAC } \\
\text { theory } \\
\text { with FE-e }\end{array}$ & $\begin{array}{c}\text { MAC } \\
\text { theory } \\
\text { with FE-f }\end{array}$ \\
\hline 1 & 2 & 23.9 & 24.3 & 24.0 & 0.4 & -1.3 & 1.00 & 1.00 \\
\hline 2 & 5 & 217.6 & 217.9 & 218.2 & 0.3 & 0.1 & 1.00 & 1.00 \\
\hline 3 & 9 & 1111.1 & 1121.4 & 1116.6 & 0.5 & -0.4 & 1.00 & 1.00 \\
\hline 4 & 10 & 1160.3 & 1163.3 & 1168.8 & 0.7 & 0.5 & 1.00 & 0.99 \\
\hline 5 & 13 & 1782.8 & 1785.7 & 1812.9 & 1.7 & 1.5 & 1.00 & 1.00 \\
\hline 6 & 15 & 2621.1 & 2696.3 & 2632.4 & 0.4 & -2.4 & 1.00 & 1.00 \\
\hline 7 & 18 & 3377.7 & 3422.4 & 3393.0 & 0.5 & -0.9 & 1.00 & 1.00 \\
\hline 8 & 19 & 3553.7 & 3560.6 & 3636.2 & 2.3 & 2.1 & 0.99 & 0.98 \\
\hline 9 & 22 & 4871.7 & 4886.4 & 5068.8 & 3.9 & 3.6 & 0.97 & 0.92 \\
\hline 10 & 23 & 5263.6 & 5429.7 & 5291.5 & 0.5 & -2.6 & 1.00 & 0.99 \\
\hline
\end{tabular}

Table 1.12.

\begin{tabular}{|c|c|c|c|c|c|c|c|c|}
\hline Mode & $\begin{array}{c}\text { FE } \\
\text { Mode }\end{array}$ & $\begin{array}{c}\text { Freq., } \\
\text { FE-g, B31 } \\
\text { (Hz) }\end{array}$ & $\begin{array}{l}\text { Freq., } \\
\text { FE-h, S4 } \\
(\mathrm{Hz})\end{array}$ & $\begin{array}{l}\text { Freq., } \\
\text { Theory } \\
(\mathrm{Hz})\end{array}$ & $\begin{array}{l}\% \text { rel. diff. } \\
\text { theory } \\
\text { with FE-g }\end{array}$ & $\begin{array}{c}\% \text { rel. diff. } \\
\text { theory } \\
\text { with FE-h }\end{array}$ & $\begin{array}{c}\text { MAC } \\
\text { theory } \\
\text { with FE-g }\end{array}$ & $\begin{array}{c}\text { MAC } \\
\text { theory } \\
\text { with FE-h }\end{array}$ \\
\hline 1 & 2 & 20.6 & 20.8 & 20.7 & 0.5 & -0.5 & 1.00 & 1.00 \\
\hline 2 & 4 & 157.5 & 157.6 & 157.7 & 0.1 & 0.1 & 1.00 & 1.00 \\
\hline 3 & 5 & 297.4 & 297.8 & 298.0 & 0.2 & 0.1 & 1.00 & 1.00 \\
\hline 4 & 7 & 624.5 & 625.8 & 625.4 & 0.1 & -0.1 & 1.00 & 1.00 \\
\hline 5 & 10 & 1115.2 & 1102.6 & 1120.0 & 0.4 & 1.6 & 1.00 & 1.00 \\
\hline 6 & 11 & 1197.0 & 1200.8 & 1200.0 & 0.3 & -0.1 & 1.00 & 0.99 \\
\hline 7 & 12 & 1760.4 & 1764.4 & 1784.9 & 1.4 & 1.1 & 0.97 & 0.99 \\
\hline 8 & 14 & 2053.9 & 2066.4 & 2067.3 & 0.6 & 0.0 & 1.00 & 1.00 \\
\hline 9 & 15 & 2528.8 & 2559.2 & 2538.8 & 0.4 & -0.8 & 1.00 & 0.99 \\
\hline 10 & 17 & 3208.8 & 3229.7 & 3226.34 & 0.5 & -0.1 & 1.00 & 0.67 \\
\hline
\end{tabular}




\section{Figure Captions}

Figure 1. Indication of axis orientation and displacements for (a) the primary beam, (b) the secondary beam.

Figure 2. Characteristic mode shapes of two motions for $1^{\text {st }}$ set of dimensions (a) in-plane bending motion for the first orientation of the secondary beam (b) out-of-plane motion for the first orientation of the secondary beam (c) in-plane bending motion for the transversal orientation of the secondary beam, (d) out-of-plane motion for the transversal orientation of the secondary beam.

Figure 3. $10^{\text {th }}$ Mode shape in in-plane bending for (a) the $1^{\text {st }}$ model, (b) the $2^{\text {nd }}$ model, (c) the $3^{\text {rd }}$ model, (d) the $4^{\text {th }}$ model.

Figure $4.10^{\text {th }}$ Mode shape for out-of-plane motions (a) $1^{\text {st }}$ model bending, (b) $1^{\text {st }}$ model torsion, (c) $2^{\text {nd }}$ model bending, (d) $2^{\text {nd }}$ model torsion.

Figure $5.10^{\text {th }}$ Mode shape for out-of-plane motions (a) $3^{\text {rd }}$ model bending, (b) $3^{\text {rd }}$ model torsion, (c) $4^{\text {th }}$ model bending, (d) $4^{\text {th }}$ model torsion. 


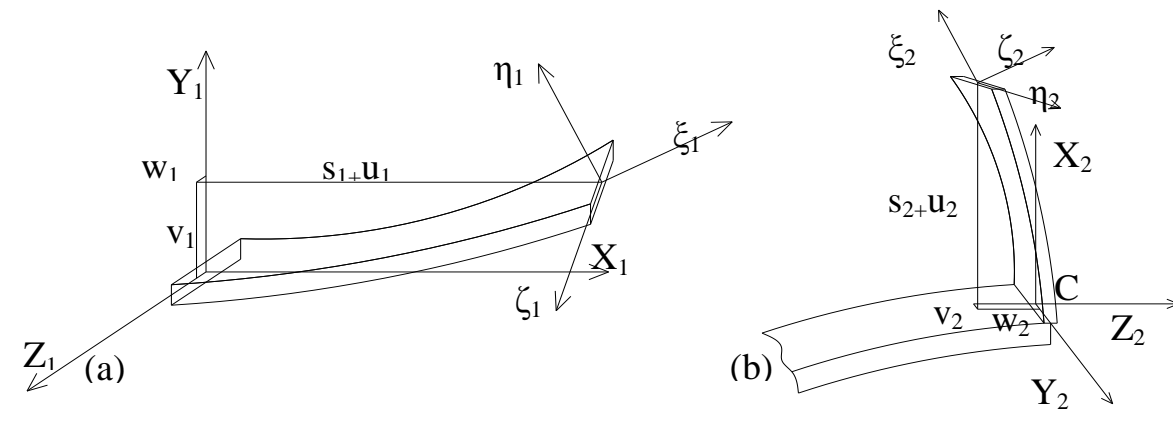

Figure 1.

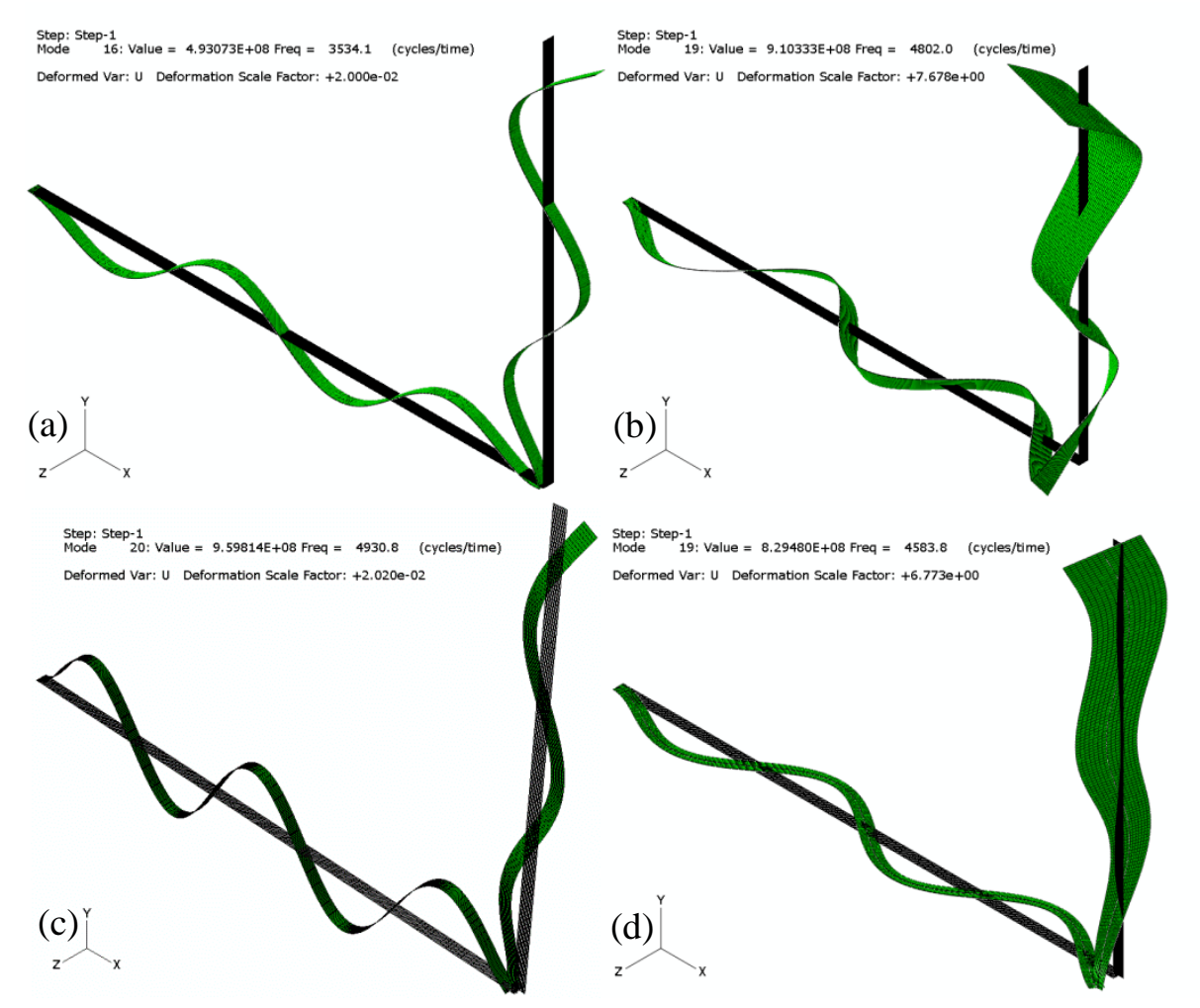

Figure 2 

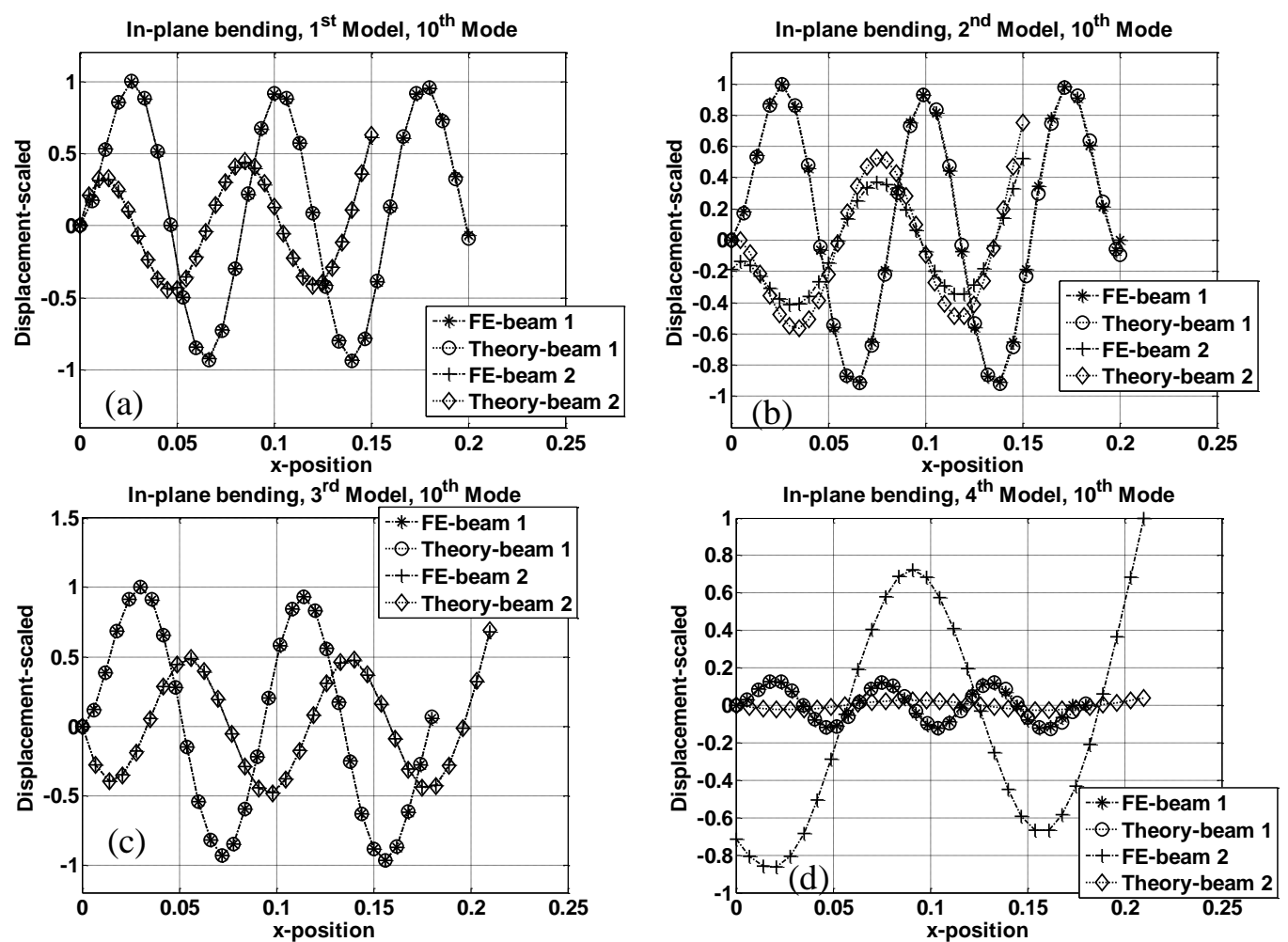

Figure 3. 

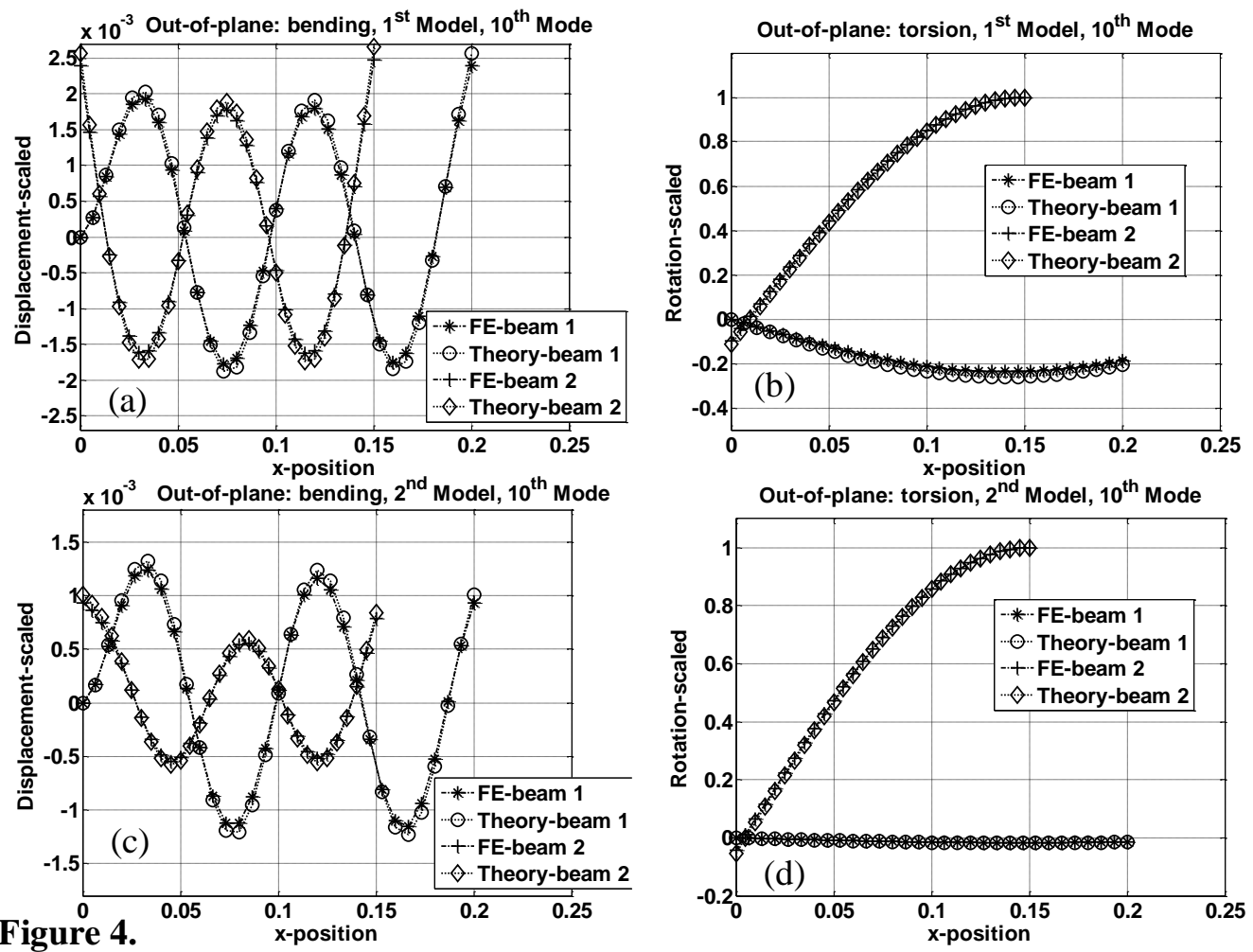

Figure 4. 

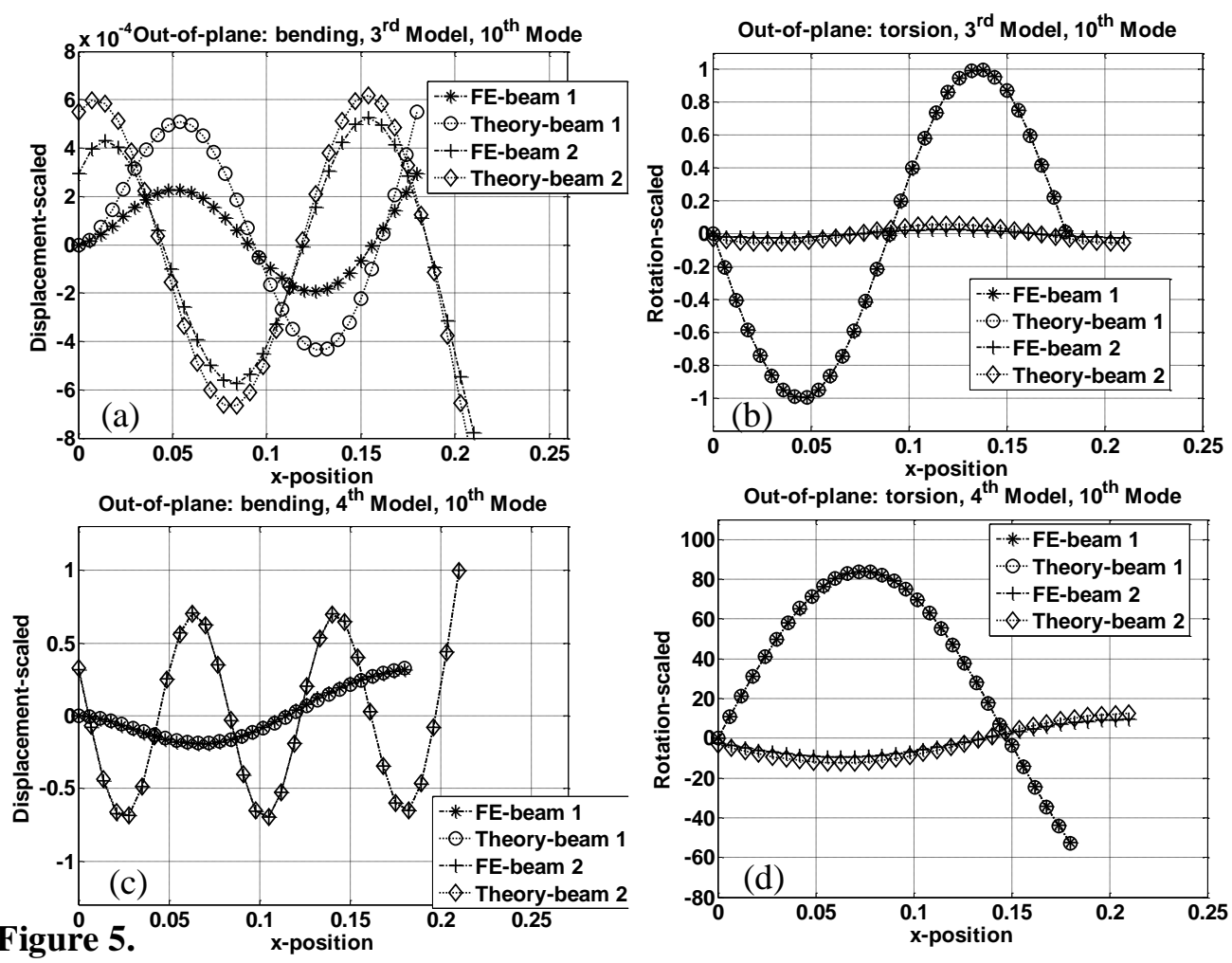